\title{
Visuotopic Organization of Macaque Posterior Parietal Cortex: A Functional Magnetic Resonance Imaging Study
}

\author{
Michael J. Arcaro, ${ }^{1,2}$ Mark A. Pinsk, ${ }^{1,2}$ Xin Li, ${ }^{2}$ and Sabine Kastner ${ }^{1,2}$ \\ ${ }^{1}$ Department of Psychology and ${ }^{2}$ Princeton Neuroscience Institute, Princeton University, Princeton, New Jersey 08540
}

\begin{abstract}
Macaque anatomy and physiology studies have revealed multiple visual areas in posterior parietal cortex (PPC). While many response properties of PPC neurons have been probed, little is known about PPC's large-scale functional topography-specifically related to visuotopic organization. Using high-resolution functional magnetic resonance imaging at $3 \mathrm{~T}$ with a phase-encoded retinotopic mapping paradigm in the awake macaque, a large-scale visuotopic organization along lateral portions of PPC anterior to area V3a and extending into the lateral intraparietal sulcus (LIP) was found. We identify two new visual field maps anterior to V3a within caudal PPC, referred to as caudal intraparietal-1 (CIP-1) and CIP-2. The polar angle representation in CIP-1 extends from regions near the upper vertical meridian (that is the shared border with $\mathrm{V} 3 \mathrm{a}$ and dorsal prelunate) to those within the lower visual field (that is the shared border with CIP-2). The polar angle representation in CIP-2 is a mirror reversal of the CIP-1 representation. CIP-1 and CIP-2 share a representation of central space on the lateral border. Anterior to CIP-2, a third polar angle representation was found within LIP, referred to as visuotopic LIP. The polar angle representation in LIP extends from regions near the upper vertical meridian (that is the shared border with CIP-2) to those near the lower vertical meridian. Representations of central visual space were identified within dorsal portions of LIP with peripheral representations in ventral portions. We also consider the topographic large-scale organization found within macaque PPC relative to that observed in human PPC.
\end{abstract}

\section{Introduction}

Multiple visual areas within and adjacent to the intraparietal sulcus have been identified in posterior parietal cortex (PPC) of macaque monkeys (Pandya and Seltzer, 1982; Andersen et al., 1990) (for review, see Van Essen, 2004). Visual areas within PPC have been distinguished based on their cytoarchitecture and myeloarchitecture as well as their connectivity patterns, including the ventral and dorsal lateral intraparietal areas (LIPv/d), and two cortical zones, dorsal prelunate (DP), and lateral occipital parietal (LOP), also referred to as caudal intraparietal (CIP). Identifying basic functional properties of these regions, such as topographic large-scale organization, may prove useful in further parcellating PPC into functional units, as it has been for other parts of the macaque visual system (Van Essen and Zeki, 1978; Maguire and Baizer, 1984; Desimone and Ungerleider, 1986) (for review, see Gattass et al., 2005).

Physiology and functional brain imaging studies have provided some evidence in support of topographic large-scale organization within PPC. Specifically, dorsal portions of area DP have been shown to exhibit spatially specific representations of the visual field (Fize et al., 2003; Heider et al., 2005), though no

Received June 25, 2010; revised Nov. 22, 2010; accepted Dec. 2, 2010.

This work was supported by National Institutes of Health Grants P50MH062196, R01-EY017699, R01MH064043, R21EY021078, and T90-DA022763 and National Science Foundation Grant BCS-1025149. M.J.A. and M.A.P. contributed equally to this study. We thank B. Singer for providing code for Fourier analyses and U. Hasson, Y. Saalmann, and N. Turk-Browne for helpful comments.

Correspondence should be addressed to Michael J. Arcaro, Department of Psychology, Princeton University, Green Hall, Princeton, NJ 08544. E-mail: marcaro@princeton.edu.

DOI:10.1523/JNEUROSCI.3334-10.2011

Copyright $\odot 2011$ the authors $\quad 0270-6474 / 11 / 312064-15 \$ 15.00 / 0$ systematic map of visual space has been demonstrated thus far. A topographic map of visual space has been reported within LIP with both physiology (Blatt et al., 1990; Ben Hamed et al., 2001) and more recently functional magnetic resonance imaging (fMRI) (Patel et al., 2010). However, there were notable differences in the pattern of topographic organization described in these studies that remain unresolved. Furthermore, previous studies of visual topography within PPC have typically focused on one particular area without investigating the relations of topographic organization to neighboring cortical areas within PPC or dorsal extrastriate cortex. Therefore, it is unclear whether macaque PPC indeed lacks organized representations of the visual field outside of LIP, as recently proposed (Patel et al., 2010). Here, we sought to investigate the visual topography across PPC and dorsal extrastriate cortex using fMRI and phase-encoded retinotopic mapping to clarify its large-scale organization.

Phase-encoded retinotopic mapping along the polar angle and eccentricity dimensions with fMRI has been widely used to reveal topographic organization within the human (Sereno et al., 1995; Engel et al., 1997; Schneider et al., 2004; Brewer et al., 2005; Hagler and Sereno, 2006; Larsson and Heeger, 2006; Kastner et al., 2007; Swisher et al., 2007; Konen and Kastner, 2008; Arcaro et al., 2009) and also the macaque visual system (Brewer et al., 2002; Kolster et al., 2009). Specifically, such topographic mapping enables the simultaneous investigation of visuotopic organization across a large region of cortex, thereby allowing the visuospatial map of an individual area to be anchored in the framework of the topographic organization across multiple surrounding areas. We investigated the representation of visual space across PPC and dorsal extrastriate cortex in awake macaque monkeys trained to 
maintain fixation for extended periods of time, and identified multiple topographically organized areas. Our results confirm the visuotopic organization within area V3a and the adjacent DP zone. Importantly, we report two new visuotopically organized areas within the caudal PPC, which we refer to as CIP-1 and CIP-2, and clarify the visuotopic organization within LIP. Last, we compare the visuotopic organization within PPC between macaque monkeys and humans.

\section{Materials and Methods}

\section{Subjects}

Two adult male macaque monkeys (Macaca fascicularis) weighing 4-6 $\mathrm{kg}$ participated in the study. All procedures were approved by the Princeton University Animal Care and Use Committee and conformed with National Institutes of Health guidelines for the humane care and use of laboratory animals.

\section{Surgical and training procedures}

Each animal was surgically implanted with a plastic head bolt for restraining the head by using ceramic screws and dental acrylic. All surgical procedures were performed under strictly aseptic conditions and under general anesthesia with isoflurane (induction $2-4 \%$, maintenance $0.5-$ $2 \%)$ following preanesthetic medication with atropine $(0.08 \mathrm{mg} / \mathrm{kg}$, i.m.), ketamine (2-10 mg/kg, i.m.), and acepromazine ( $1 \mathrm{mg} / \mathrm{kg}$, i.m.). The animals were treated postsurgically with antibiotics (e.g., Baytril, 2.5 $\mathrm{mg} / \mathrm{kg}$, i.m.) and analgesics (e.g., buprenorphine, $0.01 \mathrm{mg} / \mathrm{kg}$, i.m.), and wound margins of skin surrounding the implant were cleaned regularly.

Monkeys were placed prone in an MR-compatible primate chair in a sphinx-like position, with their heads erect and fixed in a head-holding apparatus (Pinsk et al., 2005). The animals were acclimated to the scanner environment through the use of a mock training environment. Monkeys were trained to fixate on a small $\operatorname{dot}\left(0.5^{\circ}\right.$ diameter $)$ at the center of a display screen by using an infrared eye tracking system sampling at a 60 $\mathrm{Hz}$ refresh rate (Model 504, Applied Science Laboratories). The nominal accuracy of the ASL LRO model is $0.5^{\circ}$. By providing the animals with regular juice rewards (PHD 2000, Harvard Apparatus) while they maintained fixation within a $4^{\circ} \times 4^{\circ}\left(2^{\circ}\right.$ to each side of the fixation point $)$ square window and systematically increasing the frequency of their juice reward ( 2.5 to $1 \mathrm{~s}$ in $500 \mathrm{~ms}$ steps), the animals were trained to maintain fixation for several minutes. Further details regarding surgery and training procedures have been given by Pinsk et al. (2005).

\section{Visual stimulus display and control}

Visual stimuli were projected from a single-lamp three-chip LCD projector (6500 ANSI lumens, 2000:1 contrast ratio; Christie LX650, Christie Digital Systems) outside the scanner room onto a translucent screen located at the end of the scanner bore. Monkeys viewed the projection screen directly; the total path length from eye to screen was $\sim 60 \mathrm{~cm}$. The screen subtended $30^{\circ}$ of visual angle in the horizontal and vertical dimensions. Eye tracking at the scanner was performed with a $60 \mathrm{~Hz}$ long-range optics system located outside the bore that recorded eye position through a small aperture $\left(0.75^{\circ}\right.$ diameter) in the display screen (Model LRO, Applied Science Laboratories). The stimulus presentation, eye movement tracking, and reward delivery were synchronized to the beginning of each scan using a trigger pulse from the scanner, and were controlled via a PC computer using Presentation software (Neurobehavioral Systems). During all scans, monkeys performed a fixation task, as described earlier.

\section{Visual stimuli and experimental design}

Polar angle measurements. To obtain polar angle representations, visual stimuli consisted of a wedge that rotated either clockwise or counterclockwise around a central fixation point. The wedge spanned $1-15^{\circ}$ in eccentricity with an arc length of $45^{\circ}$ and moved at a rate of $9 \mathrm{deg} / \mathrm{s}$. The wedge consisted of a colored checkerboard with each check's chromaticity and luminance alternating at a flicker frequency of $4 \mathrm{~Hz}$ [for details, see Swisher et al. (2007) and Arcaro et al. (2009)]. A transparent wedge within a dark foreground rotated around a central fixation. The underlying checkerboard was only visible through the transparent wedge, giv- ing the appearance of a rotating checkerboard wedge. Each run consisted of four cycles of $40 \mathrm{~s}$ each, and runs alternated between clockwise and counterclockwise wedge rotation. Twenty runs were collected for monkey M1, and thirty runs were collected for monkey M2, in 8 and 14 scanning sessions, respectively. For both monkeys, half of the runs were in the clockwise direction and the other half were in the counterclockwise direction.

Eccentricity measurements. To obtain eccentricity representations, visual stimuli consisted of an annulus that expanded around a central fixation point. The duty cycle of the annulus was $12.5 \%$; that is, any given point on the screen was within the annulus for only $12.5 \%$ of the time. The annulus increased on a logarithmic scale over time in size and rate of expansion to approximately match the cortical magnification factor (Horton and Hoyt, 1991). The outer part of the ring expanded to a maximum eccentricity of $16.875^{\circ}$, to ensure that the whole visual display $\left(1-15^{\circ}\right)$ was stimulated for an equal amount of time, before returning to the foveal origin. The ring consisted of a colored checkerboard with each check's chromaticity and luminance alternating at a flicker frequency of $4 \mathrm{~Hz}$ [for details, see Swisher et al. (2007) and Arcaro et al. (2009)]. A transparent annulus within a dark foreground layer expanded around a central fixation point. The underlying checkerboard was only visible through the transparent annulus, giving the appearance of an expanding checkerboard ring. Twenty runs were collected for monkey M1, and thirty runs were collected for monkey $\mathrm{M} 2$, in 5 and 14 scanning sessions, respectively.

\section{Data acquisition}

Data were acquired in both species with a $3 \mathrm{~T}$ head-dedicated scanner (Magnetom Allegra; Siemens). A $12 \mathrm{~cm}$ transmit/receive surface coil (Model NMSC-023; Nova Medical) was used for the scanning sessions during which functional images were acquired, and a $16 \mathrm{~cm}$ transmit/ receive quadrature volume coil (Model NM-016; Nova Medical) was used for a scanning session, during which high-resolution anatomical images were acquired. A whole-brain structural volume was acquired with the volume coil while the animals were anesthetized with Telazol (tiletamine/zolazepam, $10 \mathrm{mg} / \mathrm{kg}$, i.m.) in a magnetization-prepared rapid gradient echo (MPRAGE) sequence [i.e., extrasession structural: $0.5 \times 0.5 \times 0.5 \mathrm{~mm}$ resolution; field of view $($ FOV $)=128 \mathrm{~mm} ; 256 \times$ 256 matrix; repetition $(\mathrm{TR})=2500 \mathrm{~ms}$; echo time $(\mathrm{TE})=4.4 \mathrm{~ms}$; inversion time $(\mathrm{TI})=1100 \mathrm{~ms}$; flip angle $=8^{\circ} ; 20$ acquisitions]. In addition, a second whole-brain structural volume was acquired with the surface coil and the animal placed in the primate chair under anesthesia (i.e., withinsession structural: MPRAGE sequence; $0.5 \times 0.5 \times 1.0 \mathrm{~mm}$ resolution; $\mathrm{FOV}=128 \mathrm{~mm} ; 256 \times 256$ matrix; $\mathrm{TR}=2500 \mathrm{~ms} ; \mathrm{TE}=4.4 \mathrm{~ms} ; \mathrm{TI}=$ $1100 \mathrm{~ms}$; flip angle $=8^{\circ} ; 1$ acquisition). This second structural volume was acquired with the head in the same location as during the awake experimental sessions and served as an alignment reference for the higher-quality structural volume acquired with the volume coil. All other scan sessions, each lasting $\sim 1.0 \mathrm{~h}$, were performed with the animals awake.

For awake scanning sessions, an optimized multiecho gradient echo sequence was used (ME-EPI sequence; $1.5 \times 1.5 \mathrm{~mm}$ in-plane resolution; 26 axial slices; $1.5 \mathrm{~mm}$ slice thickness, no interslice gap; FOV $=120 \mathrm{~mm}$; matrix $=80 \times 80 ; \mathrm{TR}=2500 \mathrm{~ms} ; \mathrm{TE}=26 \mathrm{~ms}$; flip angle $=80^{\circ}$; bandwidth $=$ $2500 \mathrm{~Hz}$ per pixel). This sequence permits the acquisition of data at several echo times, under reversed gradient readouts, thereby allowing for simultaneous estimation of the magnetic field, resulting in reduced image distortions with partial recovery of susceptibility-induced signal loss (Pinsk et al., 2008, 2009). The slice prescription covered the entire brain.

\section{Data analysis}

Data were analyzed using AFNI (Cox, 1996) (http://afni.nimh.nih. gov/afni/), SUMA (http://afni.nimh.nih.gov/afni/suma/), Matlab (The MathWorks), and FreeSurfer (Dale et al., 1999; Fischl et al., 1999) (http://surfer.nmr.mgh.harvard.edu/). Scanning runs during which the animal refused to fixate or moved its body were easily discernible in the eye traces and EPI images. Deviations several orders of magnitude greater than the mean variance were apparent in EPI volumes where the animal moved. Due to the nature of the analyses, these runs were discarded. A 
rigid motion-correction procedure (Cox and Jesmanowicz, 1999) was performed to a reference EPI volume that was acquired during the anesthetized structural scanning session when the animal was in the primate chair. The motion-corrected data were projected onto cortical surface reconstructions created with FreeSurfer from the extrasession structural scans that were aligned to each monkeys' within-session structural. All voxels that fell between the gray and white matter boundaries were mapped to the surface. The units of data that were projected to the surface are referred to as nodes. Given that there is not a one-to-one correspondence between nodes and voxels, the value of each node was calculated by taking a weighted average of all the voxels based on the spatial overlap within each node. All subsequent analysis procedures for the visuotopy datasets (e.g., Fourier analysis) were performed on the surface-mapped data, which favorably restricts analyses to data that are primarily within the gray matter, since white matter voxels do not get mapped onto the surface. Surface projections introduce a small degree of spatial smoothing to the data. Since the degree of smoothness from surface projections can vary across cortex, the data were spatially filtered using a Gaussian filter to a maximum smoothness of $2 \mathrm{~mm}$ full-width at half-maximum (FWHM) (by estimating the FWHM before spatial filtering), ensuring uniformity across the surface and maintaining spatial specificity while increasing the signal-to-noise ratio (SNR) (Chung et al., 2005). Surface-based spatial filtering has been shown to increase both sensitivity and spatial accuracy of blood oxygenation level-dependent (BOLD) signal sources (Jo et al., 2007, 2008). Surface size estimates were measured using SUMA tools that calculate the volume of all nodes that fall in between the white matter and pial surface layers.

Volumes acquired during the blank periods at the start and end of each run were discarded. A Fourier analysis was used to identify spatially selective surface nodes by the polar angle and eccentricity stimuli (Bandettini et al., 1993; Engel et al., 1994). For each node of the surface, the amplitude and phase- the temporal delay relative to the stimulus onset- of the harmonic at the stimulus frequency were determined by a Fourier transform of the mean time series of the node. To correctly match the phase delay of the time series of each node to the phase of the wedge/ring stimuli, and thereby localize the region of the visual field to which the underlying neurons responded best, the response phases were corrected for the hemodynamic lag (5s). The counterclockwise runs were then reversed to match the clockwise runs and averaged together for each node. To quantify the reliability of phase estimates across runs, the variance of a mean phase across cycles was determined for each node. A jack-knifing method in which phase estimates were calculated from $n-$ 2 cycles (eliminating one clockwise and one counterclockwise cycle per calculation for polar angle runs) across all runs was used to determine the SE of phase estimates [for a similar application, see Hansen et al. (2007) and Arcaro et al. (2009)]. A grand mean phase estimate was calculated from the average of each of these phase estimates along with the SE to account for variance across estimates for each node. The SE was then converted into seconds per cycle.

Statistical maps were thresholded at a variance of $\pm 2 \mathrm{~s}$ of the $40 \mathrm{~s}$ cycle and overlaid on cortical surface reconstructions. The pattern and significance of activation approximately compares to a statistical threshold of $p<0.01$ (uncorrected for multiple comparisons, derived from the $F$-ratio that was calculated from the Fourier transform). When displaying phase estimates, a 20-point color scale was assigned to the polar angle datasets with each color representing $18^{\circ}$ visual angle, and a 10-point color scale was assigned to the eccentricity datasets with each color representing $1.5^{\circ}$ eccentricity. While activations were found in both ventral and dorsal striate and extrastriate cortex, ventral regions are outside the scope of this report, and we will focus our analysis on dorsal extrastriate cortex, i.e., brain regions located along the lunate sulcus and in PPC. Contiguous clusters of spatially selective nodes within this anatomical region that showed a systematic representation of visual space in polar or eccentricity coordinates were defined as regions of interest (ROIs). Borders between ROIs were manually identified by the primary author based on reversals in the systematic representation of visual space, particularly with respect to polar angle. Eccentricity representations were evaluated to ensure that phase progressions were essentially orthogonal (nonparallel) to the polar angle phase progression. Prior physiology and fMRI reports were used to guide the identification of borders for previously established visuotopically organized areas. All three authors subsequently assessed the borders between ROIs independently. Surface analyses of these ROIs were performed using AFNI and MATLAB. To evaluate the progression of phase values within each visual area and across PPC, response phase was analyzed as a function of distance on the surface by drawing small line segments that run parallel to the polar angle progression and perpendicular to the eccentricity progression. Phase values within a given area were then interpolated into a common space, which allowed for intersubject averaging. To estimate the representation of the visual field for each ROI, the polar angle phases for each surface node were binned into 20 different segments of the visual field and plotted in a polar graph as a function of percentage of the visual field coverage. To compute the representation of the visual field for each visual area as a function of polar angle, the visual field was divided into four sectors: contralateral and ipsilateral, as well as upper and lower quadrants. The number of nodes within each sector was tallied and divided by the total number of nodes in each area to derive a mean representation for each subject. Data were collapsed across hemispheres and averaged across monkeys to derive a group mean average. $t$ tests were used to assess statistical significance.

To quantitatively compare the phase alignment between clockwise and counterclockwise polar angle datasets for each monkey, we calculated alignment indices and correlation coefficients for each visual area. The alignment index (AI) was defined as Alignment Index $=1-|\Delta \phi| / \pi$, where $\Delta \phi$ is the difference between the polar angle phase for the clockwise and counterclockwise runs (for further details, see Sereno and Huang, 2006). Only nodes that had phase values for both datasets thresholded at $\pm 3 \mathrm{~s}$ variance were included in the analysis. AIs were calculated for both polar angle measurements within each ROI on a node-by-node basis. The distribution of AI values within an ROI were plotted for single subjects in a histogram. AI distributions peaking at or near 1 indicate that the two datasets were in good alignment (i.e., when the polar angle at a vertex is identical in the two datasets). In contrast, for two uncorrelated datasets the distribution of AIs is a shallow linear ramp starting at a count of zero at an AI of zero and ending at a small value $(2 v / n$, where $v$ is the number of vertices and $n$ is the number of bins) at an alignment of 1 (see Sereno and Huang, 2006). To perform statistical comparisons on the AIs, single-subject mean index values were derived by averaging across all index values obtained for individual nodes within an ROI. One-sample $t$ tests were conducted for each ROI between the mean index values and an index alignment value of 0.5 (representing chance). To derive a group index value, single-subject mean index values were averaged within each ROI. To further evaluate the strength of alignment between both datasets for a given ROI, the correlation between phase estimates was calculated on a node-by-node basis for each subject.

To directly compare visuotopic maps in the two monkeys, standardmesh cortical surfaces were created. Briefly, each monkey's surface was inflated and transformed into a sphere in a manner that minimized metric distortion (Fischl et al., 1999). The individual spheres were used to create a template sphere for each hemisphere, where the curvature pattern consisted of the average pattern across both monkeys. The individual spheres were nonrigidly aligned to the templates so that the curvature patterns of each monkey matched those of the template. To avoid interpolation of the fMRI data to match the warped spheres, the SUMA software package was used to create standard-mesh surfaces from the warped spheres using icosahedral tessellation and projection (Saad et al., 2004; Argall et al., 2006). The geometry of the resulting standard-mesh surfaces is identical to the individual monkey's original surface geometry, but the topology is common across both monkeys. The use of standard-mesh surfaces allowed for node-to-node correspondence across surfaces of both monkeys, so that functional data mapped onto one monkey's surface could be directly compared with data mapped on the other monkey's surface. Datasets for polar angle and eccentricity experiments were normalized for each monkey using a Fisher's $z$-score transformation. Average polar angle and eccentricity maps were derived by performing a Fourier analysis on the combined data from both monkeys. To further quantify the similarity in topography between the two monkeys, each monkey's data were also analyzed separately on their standard mesh 
surfaces and AI indices were calculated for both polar angle and eccentricity between them.

Cortical surfaces containing borders of the Lewis and Van Essen macaque F99 atlas (Lewis and Van Essen, 2000a,b; Van Essen, 2002) were converted from Caret (Van Essen et al., 2001) into FreeSurfer and aligned with the standard-mesh surface of the two monkeys. Both the borders and the cortical surface areas of regions defined by the atlas were compared to the corresponding regions defined by the averaged topography data.

\section{Eye-movement recordings}

Several analyses of the eye-tracking data acquired in each monkey during the scanning sessions were performed to confirm that the animals maintained fixation for the majority of the time during the scanning runs, and that there were no systematic differences in the eye position, in the amount of eye movement, or in the frequency of saccades, while different portions of the visual field were stimulated. In each monkey, eye positions, amounts of eye movement, and frequency of saccades were compared using repeated-measures ANOVAs to test for main effects of stimulus location on these measures. Individual comparisons between the stimulus locations were performed using matched paired $t$ tests. Significant pairwise comparisons $(p<0.05)$ are reported before correction for multiple comparisons, and comparisons that remained significant after Bonferroni correction ( $\alpha$ level of 0.01 ) are also reported. Effect sizes for significant pairwise comparisons are reported using Cohen's $d$ (for further details, see supplemental Methods and supplemental Fig. 1, available at www.jneurosci.org as supplemental material).

\section{Results}

Polar angle and eccentricity maps in occipital and parietal cortex

The polar angle and eccentricity components of visuotopic maps were measured for the central $15^{\circ}$ of the visual field using a smoothly rotating wedge stimulus and an expanding annulus stimulus, respectively, in monkeys trained to maintain fixation for several minutes (Pinsk et al., 2005).

Bilateral activations within striate and extrastriate cortex were found in both hemispheres of both monkeys for polar angle and eccentricity mapping studies. For the polar angle measurements, activations within each hemisphere were mainly confined to the contralateral hemifield. Individual activation maps of polar angle and eccentricity are shown overlaid on inflated surface reconstructions for the left ( $\mathrm{LH})$ and right $(\mathrm{RH})$ hemispheres for monkey M1 (Fig. 1) and monkey M2 (Fig. 2). For each surface node, the variance of the phase estimates across runs was calculated using a jack-knifing method (see Materials and Methods), and the threshold was chosen to only include data with a variance of $\pm 2 \mathrm{~s}$ per $40 \mathrm{~s}$ cycle. The color of each surface node was determined by the phase of its response and indicates the region of the visual field to which the surface node was most responsive. For the polar angle component (see Figs. $1 A, 2 A, 4 A, 6 A$, 8; supplemental Fig. 5, available at www.jneurosci.org as supplemental material), the upper visual field (UVF) is denoted in red-yellow, the horizontal meridian (HM) in green, and the lower visual field (LVF) in blue. Area boundaries that are formed by reversals in polar angle phase progression at either the UVF or LVF near the vertical meridian (VM) are indicated with dotted and dashed lines, respectively. For the eccentricity measurements (see Figs. $1 B, 2 B, 6 B$ ), the central space (foveal and parafoveal) is denoted in red/orange and the periphery in blue.

We found consistent and distinct visuotopically organized cortical areas within striate and extrastriate cortices in both monkeys. Several of these areas have been previously reported in monkey fMRI studies (Brewer et al., 2002; Fize et al., 2003; Kolster et al., 2009): V1, V2, V3, V3a, V4d, V4t, MT, MST, and FST. Anterior and lateral to $\mathrm{V} 3 \mathrm{a}$, we identified an additional visuotopic area
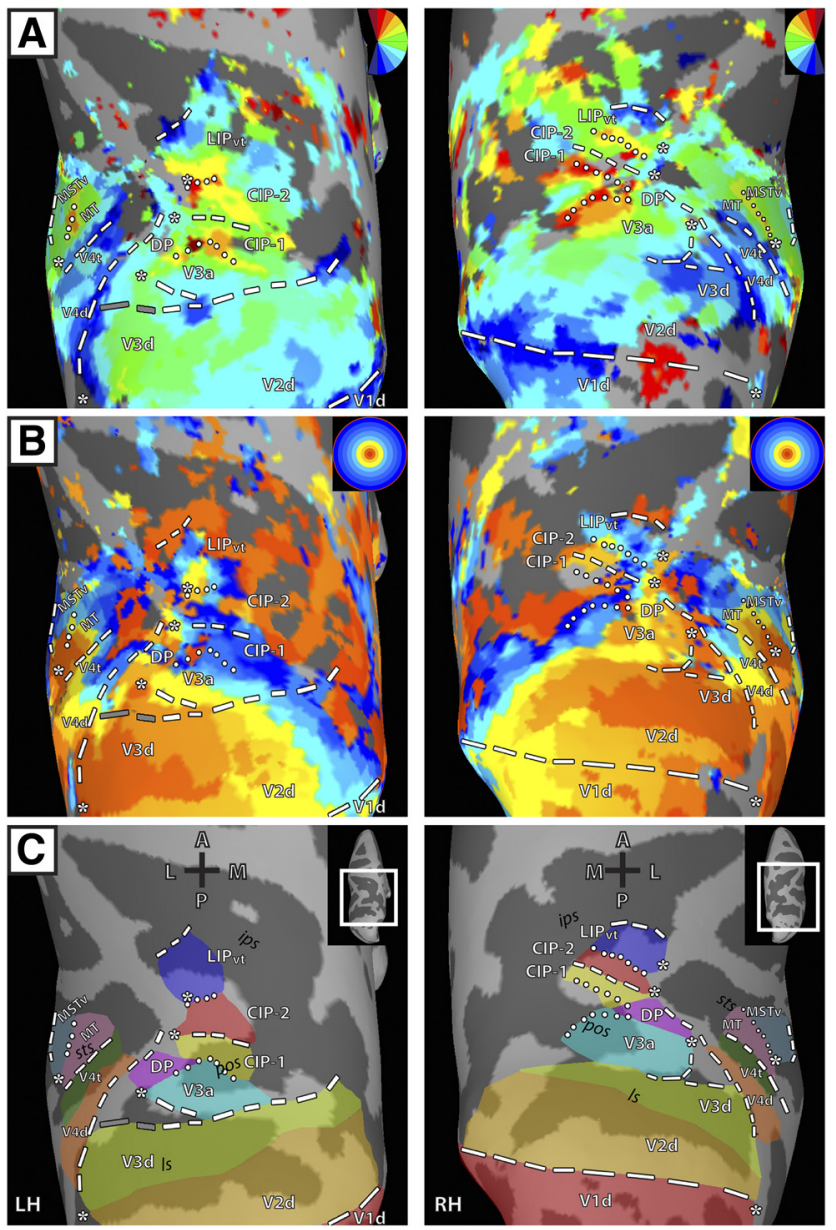

Figure 1. Polar angle and eccentricity maps in dorsal visual cortex for left and right hemispheres of M1. Inflated surface reconstructions of dorsal occipital and parietal cortex for M1. The left panel shows the topography for the LH and the right panel shows the topography for the RH. A, Polar angle maps for M1. The color code depicts the phase of the fMRI response and indicates the region of the visual field to which the surface node responds best. White lines denote area boundaries formed by phase angles at or close to the upper (dotted) or lower (dashed) vertical meridian. The gray dashed lines denote the discontinuity in the anterior border of area V3d. The surfaces are color coded such that the boundary between the dark gray and light gray represents the points of lowest curvature on the cortex with the midpoint across the dark gray region representing the fundus of the sulcal convexity and the midpoint across the light gray region representing the crown of the gyral convexity. Asterisks indicate representations of central space. Maps were thresholded at \pm 2 s per cycle SEM variance (see Materials and Methods). $\boldsymbol{B}$, Eccentricity maps for M1. The color code indicates phase of the $\mathrm{AMRI}$ response and the region of the visual field to which the surface node responds best. $C$, Schematic borders of defined topographic regions overlaid on inflated surfaces to relate the functionally defined areas and the underlying anatomy. A, Anterior; P, posterior; M, medial; L, lateral; Is, lunate sulcus; pos, parieto-occipital sulcus; sts, superior temporal sulcus; ips, intraparietal sulcus.

along the dorsal prelunate gyrus, DP (Andersen et al., 1990; Heider et al., 2005). Anterior and adjacent to V3a and DP, we identified two previously not described visuotopic areas within the caudal, lateral PPC, which we refer to as CIP-1 and CIP-2. Anterior and adjacent to CIP-2, we found another visuotopic area within LIP. We also observed significant phase activity in neighboring cortex; however, the topography was more variable and therefore no consistent visuotopic organization could be identified across the four hemispheres. Below, we provide a brief description of the previously reported areas and a more detailed one of the newly found areas.

Analysis of eye position indicated that both monkeys maintained gaze within the specified fixation window during both 

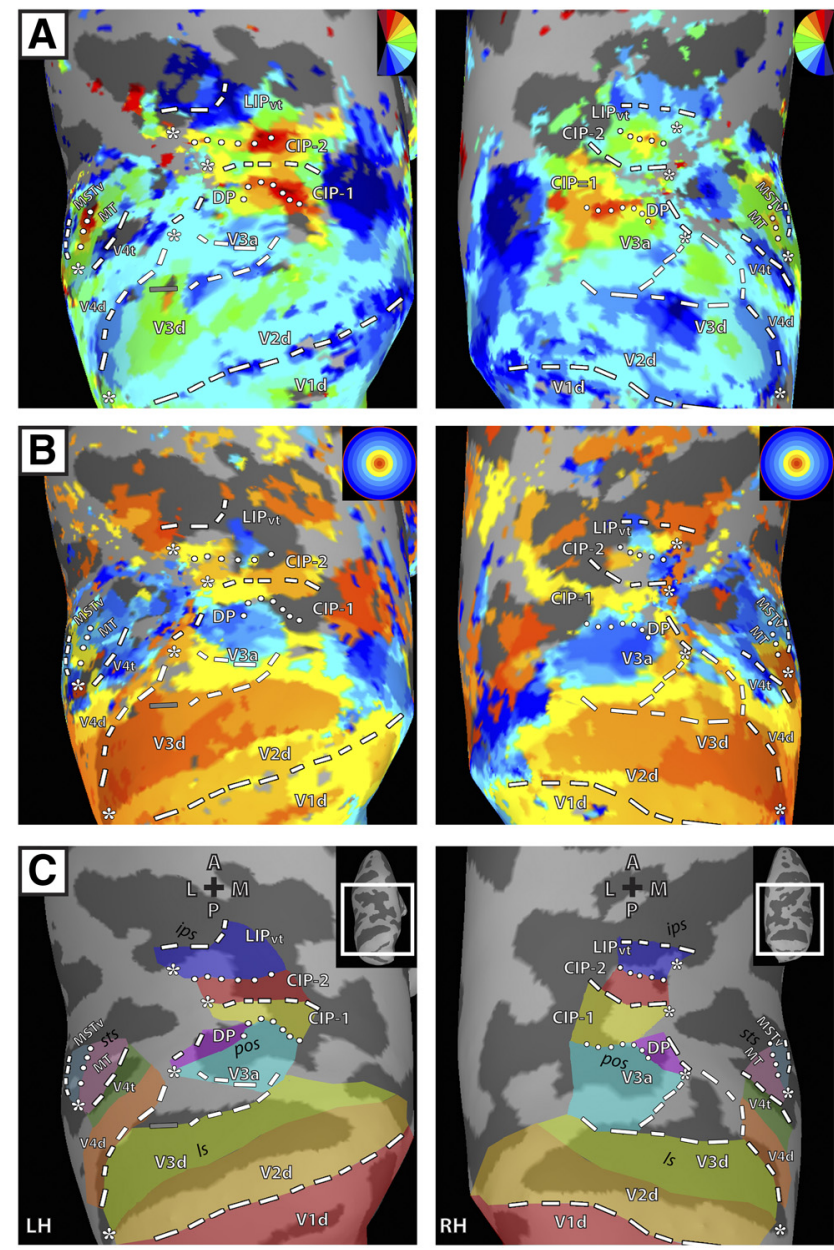

Figure 2. Polar angle and eccentricity maps in dorsal visual cortex for left and right hemispheres of M2. Inflated surface reconstructions of dorsal occipital and parietal cortex of M2. All conventions and abbreviations are as in Figure 1.

polar angle and eccentricity mapping, and that there were no significant shifts in gaze with the stimulus position during the experiments. Furthermore, analyses of eye movements and saccade frequency indicated that neither monkey had a tendency to make significantly more eye movements for any particular stimulus position (for further details, see supplemental Methods and supplemental Fig. 1, available at www.jneurosci.org as supplemental material).

\section{Areas V1, V2, and V3}

In agreement with previous reports (Daniel and Whitteridge, 1961; Zeki, 1969; Van Essen et al., 1984; Brewer et al., 2002; Fize et al., 2003), visual areas V1, V2, and V3 were identified in each hemisphere of both monkeys by a phase progression starting within the calcarine sulcus from an HM representation to a VM representation (Figs. 1A, $2 \mathrm{~A}$, blue color-coded phase, dashed line) that forms the border to area $\mathrm{V} 2$, and then reversing back to an HM that corresponds to the border of V2 and V3 (Figs. $1 A$, $2 A$, green, boundary not drawn).

Within dorsal extrastriate cortex, a phase progression was identified from the HM border of V2d/V3d to an LVM representation (Figs. $1 \mathrm{~A}, 2 \mathrm{~A}$, blue color-coded phase, dashed line) corresponding to the anterior border of V3d. In two hemispheres, the anterior LVM border of V3d was discontinuous within the lunate sulcus with a representation near the HM in between LVM representations, as seen in the LH of M1 (Fig. $1 A$, left column), but continuous in the other hemispheres as seen in the RH of M1 (Fig. $1 A$, right column). These results are in agreement with previous physiology studies that have reported two variants of the anterior V3d border, one forming a continuous representation of the LVM, and the other forming a discontinuous representation of the LVM with HM representations intermixed [for examples of the two variants, see Gattass et al. (1988), their Figs. 5, 22].

All three areas share a representation of central space within the anterior extent of the calcarine sulcus with representations of the periphery extending both dorsally and ventrally approximately perpendicular to the polar angle phase progressions (Figs. $1 B, 2 B)$.

\section{Areas V3a and DP}

A representation of the contralateral visual field including both the LVF and UVF was identified within the lunate sulcus and parieto-occipital sulcus (POS) of each hemisphere, consistent with known topography of area V3a (Van Essen and Zeki, 1978; Gattass et al., 1988; Brewer et al., 2002; Fize et al., 2003). The posterior, lateral border of V3a was identified by a LVF representation within the dorsal, anterior bank of the lunate sulcus and along the cortex separating the lunate sulcus from the POS (Figs. $1 A, 2 A$, blue color-coded phase, dashed line). In all hemispheres, the posterior portion of the LVF representation was continuous with the LVM border of V3d, but separated from the V3d border, as it extended in an anterior direction to the prelunate gyrus. A polar angle phase progression of contralateral space was identified extending from this LVF representation in a caudal/lateralrostral/medial direction to an UVF representation within the POS (Figs. $1 A, 2 A$, red color-coded phase, dotted line). A representation of the central space was found within the prelunate gyrus, anterior and lateral to portions of cortex that split the lunate sulcus and POS (Figs. $1 B, 2 B$, asterisk). The peripheral representations of the eccentricity map extended ventrally into both the posterior and anterior POS (Figs. $1 \mathrm{~B}, 2 \mathrm{~B}$ ). The anatomical extent of $\mathrm{V} 3 \mathrm{a}$ was consistent between hemispheres and monkeys, as is shown on the surface (Figs. $1 C, 2 C$ ) as well as within the volume (Fig. 3; supplemental Figs. 2, 3, available at www. jneurosci.org as supplemental material). The borders for $\mathrm{V} 3 \mathrm{a}$, in Horsley Clarke stereotaxic coordinates, extended from -6.0 to -12.5 [anterior-posterior $(\mathrm{A}-\mathrm{P})$ ], +19.5 to +25.5 [inferior-superior $(\mathrm{I}-\mathrm{S})]$, and +7.0 to +16.0 [medial-lateral $(\mathrm{M}-\mathrm{L})]$ (Table 1 ). The mean surface volume estimate (measured between pial and white matter) for V3a was $45.6 \pm 3.0 \mathrm{~mm}^{3}$ (Table 2).

A LVF representation of contralateral space was identified anterior to $\mathrm{V} 3 \mathrm{a}$ along the dorsal portion of the prelunate gyrus in each hemisphere, which we tentatively label area DP (May and Andersen, 1986; Gattass et al., 1988; Fize et al., 2003; Heider et al., 2005) (but also see Maguire and Baizer, 1984). A representation of the HM was identified within the POS, adjacent and medial to this LVF representation with an UVF representation extending into area V3a located further medially within the POS (Figs. $1 A$, $2 A)$. This region was largely encompassed by a representation of central space abutting representations of central space within $\mathrm{V} 3 \mathrm{a}$, but also included cortex along the prelunate gyrus, located anterior and medial to the central space, that appeared to be distinguished from the topography of V3a (Figs. $1 B, 2 B$ ). The anterior portion of $\mathrm{V} 3$ a roughly encompassed the posterior half of the central space and the posterior portion of DP encompasses the anterior half of the central space. No clear eccentricity progression was apparent, though consistent representations of the periphery were observed in this region anterior and medial to the central space (e.g., Fig. 2 B, LH of M2, left column). The anatom- 

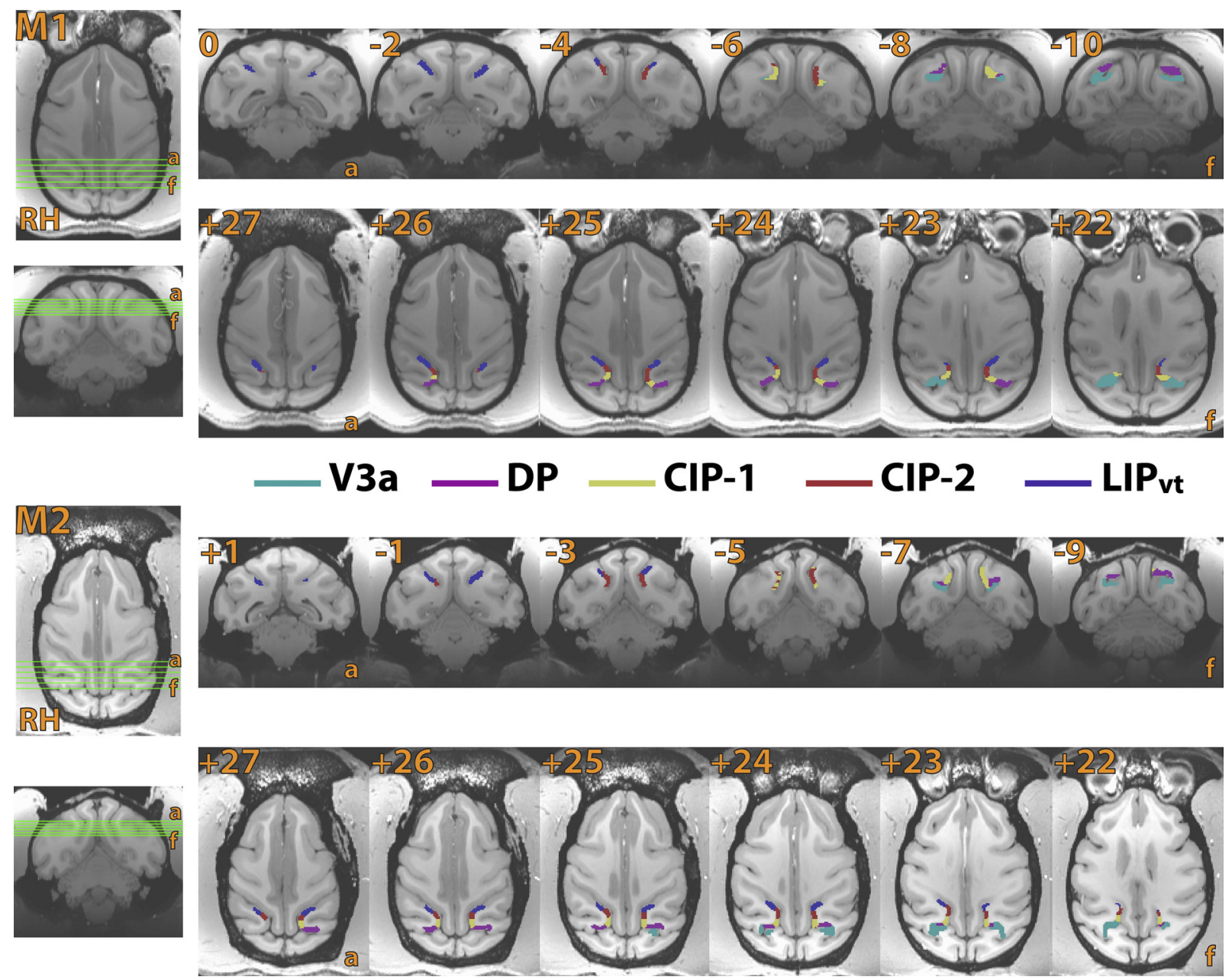

Figure 3. Area boundaries of $\mathrm{V} 3 \mathrm{a}, \mathrm{DP}, \mathrm{CIP}-1, \mathrm{CIP}-2$, and $\mathrm{LIP}$ vt of $\mathrm{M} 1$ and $\mathrm{M} 2$ in coronal and axial sections. Coronal (top) and axial (bottom) sections for monkey M1 (top) and M2 (bottom) slice segments are shown. Reference images are displayed on the left side, illustrating the coverage of each coronal and axial slice. Borders were defined on the surface, projected into the volume, and color coded by area.

Table 1. Range of Horsley Clarke coordinates for right and left hemispheres of V3a, DP, CIP-1, CIP-2, and LIP

\begin{tabular}{|c|c|c|c|c|c|c|}
\hline & \multicolumn{3}{|l|}{ Left hemisphere } & \multicolumn{3}{|l|}{ Right hemisphere } \\
\hline & $A-P$ & $I-S$ & $\mathrm{M}-\mathrm{L}$ & $A-P$ & $I-S$ & $M-L$ \\
\hline \multicolumn{7}{|l|}{ V3a } \\
\hline M1 & -6.0 to -12.0 & +19.5 to +22.5 & +7.5 to +16.0 & -6.0 to -12 & +20.0 to +23.5 & +7.0 to +15.0 \\
\hline M2 & -6.5 to -12.5 & +21.0 to +25.5 & +7.5 to +14.5 & -6.0 to -12 & +21.0 to +24.5 & +7.5 to +14.0 \\
\hline \multicolumn{7}{|l|}{ DP } \\
\hline M1 & -7.0 to -10.5 & +22.5 to +25.5 & +7.5 to +13.5 & -7.0 to -10.5 & +23.0 to +26.5 & +6.5 to +13.5 \\
\hline M2 & -6.5 to -10.5 & +22.0 to +28.0 & +6.0 to +14.0 & -7.0 to -10.0 & +22.5 to +26.0 & +7.0 to +13.0 \\
\hline \multicolumn{7}{|l|}{ (IP-1 } \\
\hline M1 & -5.5 to -9.5 & +20.0 to +25.5 & +6.5 to +10.0 & -5.0 to -8.0 & +21.5 to +26.5 & +5.5 to +9.5 \\
\hline M2 & -4.5 to -8.5 & +20.5 to +28.5 & +5.5 to +9.5 & -3.5 to -8.0 & +21.0 to +26.5 & +5.5 to +8.5 \\
\hline \multicolumn{7}{|l|}{ (IP-2 } \\
\hline M1 & -3.0 to -7.5 & +20.5 to +26.0 & +6.0 to +8.5 & -3.0 to -6.0 & +22.5 to +27.0 & +6.0 to +8.0 \\
\hline M2 & -2.5 to -5.5 & +21.5 to +28.5 & +5.5 to +8.5 & -1.0 to -5.5 & +22.0 to +27.0 & +5.5 to +8.5 \\
\hline \multicolumn{7}{|l|}{$\mathrm{LIP}_{\mathrm{vt}}$} \\
\hline M1 & +1.0 to -4.5 & +22.0 to +27.0 & +6.5 to +12.0 & +0.5 to -5.0 & +23.0 to +29.0 & +6.5 to +13.0 \\
\hline M2 & +1.0 to -3.5 & +22.5 to +29.0 & +6.0 to +11.5 & +1.5 to -4.0 & +22.0 to +28.0 & +7.0 to +12.0 \\
\hline
\end{tabular}

Table 2. Surface volume estimates (measured between pial and white matter) for V3a, DP, CIP-1, CIP-2, and LIP ${ }_{\text {vt }}$

\begin{tabular}{lrrrrrr}
\hline & \multicolumn{1}{c}{ V1 } & \multicolumn{1}{c}{ V3a } & \multicolumn{1}{c}{ DP } & CIP-1 & CIP-2 & LIP vt \\
\hline Surface volume $\left(\mathrm{mm}^{3}\right)$ & 1092.5 & 45.6 & 35.1 & 32.1 & 34.3 & 38.6 \\
SEM $\left(\mathrm{mm}^{3}\right)$ & 45.6 & 2.5 & 3.1 & 4.8 & 4.5 & 4.6 \\
\% of V1 & 100.0 & 4.2 & 3.2 & 3.0 & 3.1 & 3.5 \\
\hline
\end{tabular}

ical extent of DP was consistent between hemispheres and monkeys, as is shown on the surface (Figs. $1 C, 2 C$ ) as well as within the volume (Fig. 3; supplemental Figs. 2, 3, available at www. jneurosci.org as supplemental material). The borders for DP extended from -6.5 to -10.5 (A-P), +22.0 to +28.0 (I-S), and +6.0 to $+14.0(\mathrm{M}-\mathrm{L})($ Table 1$)$. The mean surface volume esti- 


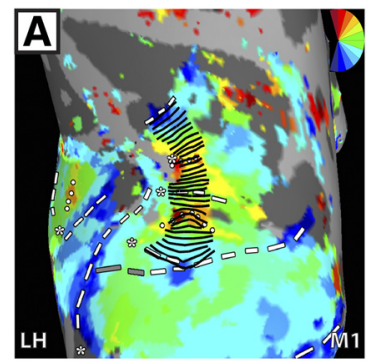

B
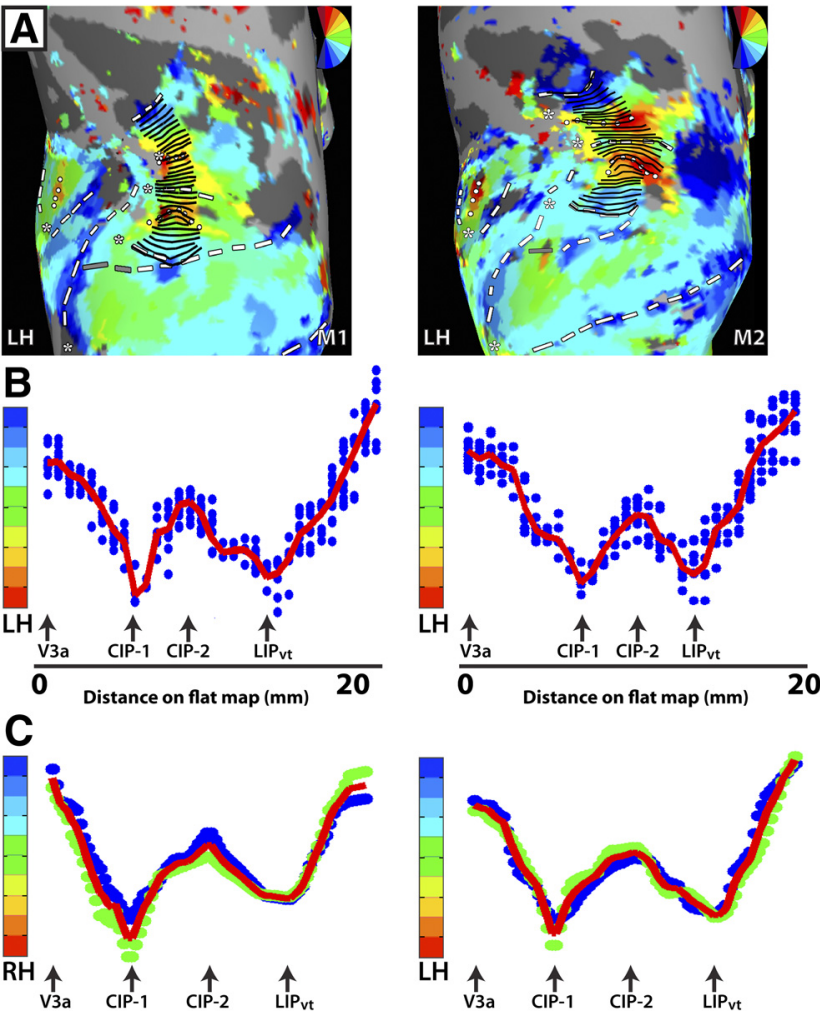

Figure 4. Analysis of topographic organization within V3a, CIP-1, CIP-2, and LIP. A, Polar angle maps of dorsal occipital and parietal cortex for the LH of M1 (left column) and M2 (right column). Response phase was analyzed as a function of distance on the surface by drawing small line segments that run parallel to the polar angle progression and perpendicular to the eccentricity progression. The line segments were successively drawn from the lateral, posterior border of V3a to the anterior border of $L I P_{v t}$. $\boldsymbol{B}$, Polar angle phase plot for $L H$ of M1 (left column) and $\mathrm{M} 2$ (right column) as a function of distance from the lateral, posterior border of $\mathrm{V} 3 \mathrm{a}$ (in $\mathrm{mm}$ ). The blue dots indicate the phase values for individual nodes located along the line segments. The red line indicates the average phase values as a function of distance on the surface. Note the smooth progression of phase values as a function of distance on the map. Importantly, the response phase reverses at the shared boundaries between adjacent areas (black arrows). $C$, Group polar angle phase plots are shown for both $\mathrm{RH}$ and $\mathrm{LH}(N=2)$. Phase values within a given area were interpolated into a common space, which allowed for intersubject averaging. The blue and green dots indicate phase values for individual subjects after interpolation. The red line indicates the group average. The smooth progression of phase values within a given area and the phase reversals at area boundaries are apparent in the group averages as well as in the individual subjects.

mates (measured between pial and white matter) for DP was $35.1 \pm 3.0 \mathrm{~mm}^{3}$ (Table 2).

To further investigate the topographic organization of V3a, the polar angle phase progression was quantified and evaluated. Small line segments were successively drawn parallel to the polar angle progression and perpendicular to the eccentricity progression (see Materials and Methods) from the posterior border of $\mathrm{V} 3 \mathrm{a}$ to the shared anterior border, as indicated by the schematic lines in Figure $4 A$. As shown in Figure $4 B$, the polar angle phase values from these line segments were plotted as a function of distance (in millimeters) from the rostral border of V3a. The blue dots indicate the phase values for individual nodes located along the line segments, and the red line indicates the average phase values as a function of the distance on the surface. Polar angle phase progressions between area borders for individual monkeys were interpolated into a common space, which allowed for group averaging (Fig. 4C). As seen in the LH of M1 and M2 (Fig. 4B), as well as in the group polar phase plot (Fig. $4 C$ ), the posterior and anterior borders of $\mathrm{V} 3 \mathrm{a}$ corresponded to the peaks of phase angles near the LVF and UVF, respectively, with a smooth progression of phase values in between.

\section{Areas CIP-1 and CIP-2}

Anterior to area $\mathrm{V} 3 \mathrm{~A}$, two polar angle phase representations of contralateral space were found in each hemisphere, spanning across the POS, posterior portions of the intraparietal sulcus (IPS), and posterior portions of the inferior parietal gyrus (at the junction of the prelunate gyrus and the inferior parietal gyrus), which we refer to as CIP-1 and CIP-2. In each hemisphere, CIP-1 was defined by a polar angle phase progression extending from the UVF representation of V3a/DP (red color-coded phase/dotted line) anterior to a phase reversal in posterior IPS (Figs. $1 A$, $2 A)$. In two of the four hemispheres, a small strip of LVF representation (blue color-coded phase, dashed) within an HM representation defined the phase reversal as seen in the RH of M2 (Fig. $2 A)$. In the other two hemispheres, the phase progression reversed within an HM representation (also indicated with dashed line). In all hemispheres, CIP-2 was defined by polar angle phase progressions extending further anterior to an UVF representation (red color-coded phase, dotted line) within the posterior portion of the IPS (Figs. 1A, 2A). The UVF representation spanned across the inferior parietal gyrus medially and into the fundus of the IPS. This region of cortex within the lateral bank of caudal IPS likely corresponds to a region of cortex that has been previously referred to as cIPS (Sakata et al., 1998), LOP (Lewis and Van Essen, 2000a,b), CIP (Taira et al., 2000), CIPS (Tsao et al., 2003), and pLIP (Kagan et al., 2010). In the RH hemisphere of $\mathrm{M} 1$, the UVF representation of CIP-1 appeared fragmented from medial portions of the UVF representation of V3a (Fig. 1A), though the remaining polar angle phase progression was consistent with the other hemispheres. The mirror reversal in phase angle representations within CIP-1 and CIP-2 is further illustrated by the pattern of phase progressions (Fig. 4).

A representation of central space was identified anterior to that of V3a, and overlapped with lateral portions of CIP-1 and CIP-2 along the lateral IPS and onto the inferior parietal lobule (Figs. $1 B, 2 B$, asterisk). The central space representations of CIP$1 / 2$ appeared continuous with the central space representations of V3a in two hemispheres as seen in the RH of M1 (Fig. $1 B$ ), but were clearly separate in the LH of M2 (Fig. $2 B$ ). The eccentricity maps lacked detail in this region of cortex. In M1, representations of the periphery extended medially into the fundus of the parietooccipital confluence and IPS (Fig. 1B). However, eccentricity representations were mostly central space and parafoveal in M2 (Fig. 2 B). The anatomical extent of CIP-1 and CIP-2 was consistent between hemispheres and monkeys, as is shown on the surface (Figs. 1C, 2C) as well as within the volume (Fig. 3; supplemental Figs. 2, 3, available at www.jneurosci.org as supplemental material). The borders for CIP-1 extended from -3.5 to $-9.5(\mathrm{~A}-\mathrm{P}),+20.0$ to +28.5 (I-S), and +5.5 to $+10.0(\mathrm{M}-\mathrm{L})$ and for CIP-2 from -1.0 to -7.5 (A-P), + 20.0 to +28.5 (I-S), and +5.5 to $+8.5(\mathrm{M}-\mathrm{L})$ (Table 1$)$. The mean surface volume estimates (measured between pial and white matter) for CIP-1 and CIP-2 were $32.1 \pm 4.8 \mathrm{~mm}^{3}$ and $34.2 \pm 4.5 \mathrm{~mm}^{3}$, respectively (Table 2).

Area LIP $_{v t}$

A representation of the contralateral visual field was observed within the lateral portion of the IPS anterior to CIP-2 in all four hemispheres, referred to as $\mathrm{LIP}_{\mathrm{vt}}$. A polar angle phase progression extended in a caudal-rostral direction from the UVF representa- 


\begin{tabular}{|c|c|c|c|c|c|}
\hline & V3a & DP & CIP-1 & CIP-2 & $\mathrm{LIP}_{\mathrm{vt}}$ \\
\hline \multicolumn{6}{|l|}{ Amplitude } \\
\hline Polar angle & $1.32(0.30)$ & $0.95(0.20)$ & $0.40(0.05)$ & $0.60(0.10)$ & $0.40(0.09)$ \\
\hline Eccentricity & $0.57(0.08)$ & $0.40(0.09)$ & $0.30(0.10)$ & $0.14(0.03)$ & $0.17(0.04)$ \\
\hline \multicolumn{6}{|l|}{ Visual field coverage } \\
\hline Contra versus Ipsi & 99 (1)\% & $94(3) \%$ & $97(1) \%$ & $97(1) \%$ & $96(1) \%$ \\
\hline Upper versus lower & $40(4) \%$ & $70(4) \%$ & $72(4) \%$ & $84(6) \%$ & $42(7) \%$ \\
\hline
\end{tabular}

Values in parentheses are SES.

tion shared by CIP-2 to a LVF representation that spanned across the lateral bank and fundus of the IPS (Figs. 1 $A, 2 A$ ). In agreement with prior reports, this phase progression within lateral IPS (Blatt et al., 1990; Ben Hamed et al., 2001) encompassed both ventral and dorsal portions of LIP with the UVF located slightly more lateral to the LVF. The mirror reversal in phase angle representations within $\operatorname{LIP}_{\mathrm{vt}}$ is further illustrated by the pattern of phase progressions (Fig. 4).

In all four hemispheres, a representation of central space was identified anterior to CIP-1 and CIP-2 along the lateral bank of the IPS (Figs. $1 B, 2 B$, asterisk). The central space appeared continuous with the representations of central space within dorsolateral portions of CIP in two hemispheres, as seen in the RH of M1 (Fig. $1 B$ ), but was separate in the other two hemispheres, as seen in the LH of M1 (Fig. $1 B$ ). In agreement with prior reports (Blatt et al., 1990; Ben Hamed et al., 2001), no clear progression of eccentricity was observed, though peripheral representations were generally observed ventromedially, along the fundus of the IPS, as seen in the RH and LH of M1 (Fig. $1 B$ ). Aside from a dorsolateral-central and ventromedial-peripheral distinction, the eccentricity maps within this region of cortex were quite coarse. The anatomical extent of $\operatorname{LIP}_{\mathrm{vt}}$ was consistent between hemispheres and monkeys, as is shown on the surface (Figs. $1 C$, $2 C$ ) as well as within the volume (Fig. 3; supplemental Figs. 2, 3, available at www.jneurosci.org as supplemental material). The borders for $\mathrm{LIP}_{\mathrm{vt}}$ extended from +1.5 to $-5.0(\mathrm{~A}-\mathrm{P}),+22.0$ to $+29.0(\mathrm{I}-\mathrm{S})$, and +6.0 to $+13.0(\mathrm{M}-\mathrm{L})$ (Table 1$)$. The mean surface volume estimates (measured between pial and white matter) for LIPvt was $38.6 \pm 4.6 \mathrm{~mm}^{3}$ (Table 2).

To evaluate the strength of the stimulus-evoked signal relative to noise in LIP $_{\mathrm{vt}}$, as well as V3a, DP, CIP-1 and CIP-2, response amplitudes were calculated as a function of temporal frequency for the polar angle and eccentricity measurements. For each monkey, the response at the stimulus frequency (SF) of four cycles was at least 2 SEs greater than the mean response across all other frequencies, demonstrating a strong link between the measured neural response and stimulus location. Temporal frequency histograms were derived for each monkey and each hemisphere and then averaged to yield group data. For both the polar angle and eccentricity data, the response at the SF ( 4 cycles) was significantly greater than noise for V3a, DP, CIP-1, CIP-2, and $\operatorname{LIP}_{\mathrm{vt}}$ (Table 3 ) (for polar angle, all $t$ values $>4.30, p$ values $<0.05$; for eccentricity, all $t$ values $>3.40, p$ values $<0.05$ ). In a complementary analysis, the time series of fMRI signals within line segments from the polar angle phase progression analysis corresponding to the UVF and LVF reversal points were averaged to derive a mean time series for each border between visuotopic areas in PPC. Consistent with the power analysis, the stimulusevoked signal in V3a, DP, CIP-1, CIP-2, and $\mathrm{LIP}_{\mathrm{vt}}$ was apparent in the time series, as demonstrated in monkey M1 (supplemental Fig. $4 A$, available at www.jneurosci.org as supplemental mate- rial). The phase offsets between UVF and LVF time series were estimated by calculating the correlations between the two time series across 10 time point offsets for each ROI and measuring the distance between the original time series and the offset time series with the highest correlation. The time series between UVF and LVF borders were out of phase with each other on average by $17 \pm .5 \mathrm{~s}$ for M1 and $17.5 \pm .79 \mathrm{~s}$ for M2. To evaluate the relation of phase between UVF and LVF borders with polar angle phase estimates, the time series were averaged between monkeys and collapsed across cycles. The modulation in fMRI signal for each area corresponded to the estimated phases from the Fourier analysis (supplemental Fig. $4 B$, available at www. jneurosci.org as supplemental material). The cycles of UVF borders (V3a/CIP-1 and CIP-2/LIP ${ }_{\mathrm{vt}}$ ) were in phase with each other and $17.5 \mathrm{~s}$ out of phase with the cycle of LVF anterior border of LIP ${ }_{\mathrm{vt}}$. The cycle of the LVF border between CIP-1 and CIP-2 was $5 \mathrm{~s}$ out of phase from the cycle of the anterior LVF border of $\mathrm{LIP}_{\mathrm{vt}}$, consistent with the phase progression analysis that demonstrated the phase progression reversal point to be closer to the HM.

Other topographic representations in surrounding cortical areas Additional polar angle and eccentricity phase estimates were observed across neighboring cortex, which are beyond the scope of the present report, but will be briefly described here to give a comprehensive account of the data.

\section{V4 complex-areas V4d and V4t}

In agreement with previous studies (Zeki, 1969; Zeki, 1980; Maguire and Baizer, 1984; Desimone and Ungerleider, 1986; Gattass et al., 1988) (also see Fize et al., 2003), visual areas V4d and V4t were identified in each hemisphere of both monkeys. A polar angle phase progression of contralateral space was identified from the LVM representation corresponding to the border between $\mathrm{V} 3 \mathrm{~d}$ and $\mathrm{V} 4 \mathrm{~d}$ to an HM representation corresponding to the border between V4d and V4t (Figs. 1A, 2A). The phase progression reversed further anterior into the ventral bank of the posterior superior temporal sulcus (STS) to an LVM representation corresponding to the border between V4t and MT/V5. The central space representation of V4d appears to be continuous with the central space representation shared by early visual areas $\mathrm{V} 1, \mathrm{~V} 2$, and V3 (Figs. $1 \mathrm{~B}, 2 \mathrm{~B}$ ). The central space representation of $\mathrm{V} 4 \mathrm{t}$ is located more laterally within the fundus of posterior STS and may be distinct from the central space representations of early visual areas (see Kolster et al., 2009).

The anterior lateral portion of the LVM representation of V3a was identified as separate from the anterior border of $\mathrm{V} 3 \mathrm{~d}$ in all hemispheres (Figs. 1 A, 2A). This resulted in a triangular region on the cortical surface in between $\mathrm{V} 3 \mathrm{a}, \mathrm{V} 3 \mathrm{~d}$, and $\mathrm{V} 4 \mathrm{~d}$. This region consistently fell within an anterior portion of the lunate sulcus that was indented into the prelunate gyrus (Figs. 1,2). This region largely represented the contralateral HM and LVF and mainly represented central portions of visual space with the representations of central space shared by V3a and DP, located adjacent and medial to this region (Figs. 1,2). The apparent phase progression within the lower visual field suggests an organized representation of at least part of the contralateral visual field. However, this region may correspond to a transition region between dorsal V4 and DP (Lewis and Van Essen, 2000a), and not to specific visual field map (s). Further, the observed topography within this region of cortex is not easily relatable to the large-scale topographic organization observed in humans as the correspondence in topographic organization of dorsal V4 and the surrounding cortex to 
humans (Larsson and Heeger, 2006; Hansen et al., 2007) is still unclear and may be significantly differ between species (Wandell et al., 2007; Kolster et al., 2010). Given the region's relatively small spatial extent and the limited spatial resolution of our methods, identifying precise topographic organization was inconclusive. A systematic investigation of this region and the surrounding cortex including V4d and V4t at higher resolution and therefore higher field strength (and possibly additional functional experiments) would be required to resolve the topography and its relation to neighboring areas, and is therefore beyond the scope of the current study.

\section{Area $7 a$}

Along the inferior parietal gyrus, lateral and adjacent to the central space representations of CIP- 1, CIP-2, and LIP $_{\mathrm{vt}}$, a representation of the contralateral LVF was evident in all hemispheres, though no clear topographic organization could be identified beyond these representations (Figs. 1,2). This region of cortex likely corresponds to area 7a (Andersen et al., 1990). This part of cortex appears to have mainly peripheral representations, but also overlaps with central space regions shared by CIP-1 and CIP-2 (Figs. 1B, 2B). Notably, Heider et al. (2005) have found coarse retinotopic organization within area 7a using optical imaging techniques. However, the specific details of the topographic organization appeared to vary from day to day. Though our results suggest the existence of some spatial topography within this region of cortex, no clearly organized map of the visual field could be identified, consistent with the interpretation that area $7 \mathrm{a}$ forgoes a static retinotopic organization for a spatial topography capable of adapting to alternating environments (Heider et al., 2005).

\section{STS — areas MT, MSTv, and FST}

Polar angle phase progressions of contralateral space were observed within posterior STS, consistent with previous studies delineating MT, MSTv, and FST (Kolster et al., 2009) [also see Gattass and Gross (1981), Van Essen et al. (1981), and Desimone and Ungerleider (1986)]. In all hemispheres, a polar angle progression was identified from the LVM representation (Figs. $1 A$, $2 A$ ) corresponding to the border between V4t and MT to an UVF representation within the lower bank of posterior STS corresponding to the border between MT and MSTv. The polar angle progression reversed back toward a LVF representation further anterior and within the fundus of the STS corresponding to the border between MSTv and FST.

Within the dorsal portion of the posterior STS, superior to MT and MST, a peripheral representation of the LVF and HM was observed in all four hemispheres (Figs. 1, 2). As suggested by Kolster et al. (2009), this anatomical area may correspond to MTp or other neighboring motion-sensitive areas (Desimone and Ungerleider, 1986). No apparent representations central space could be identified.

\section{Visual field representations}

As seen in Table 3, areas V3a, DP, CIP-1, CIP-2, and LIP represented almost exclusively the contralateral visual field (contralateral vs ipsilateral visual field: all $t_{(3)}$ values $>25$, $p$ values $<0.0001$ ). For comparison, $97 \pm 1 \%$ of nodes represented contralateral visual space in area V1. The strong laterality is further demonstrated in the polar plots of V1, V3a, DP, CIP-1, CIP-2, and $\operatorname{LIP}_{\mathrm{vt}}$ (Fig. 5). As is evident in the polar plots as well as the polar angle maps, $\mathrm{V} 3 \mathrm{a}$ and $\mathrm{LIP}_{\mathrm{vt}}$ contained roughly equivalent representations of the upper and lower quadrants of the con-
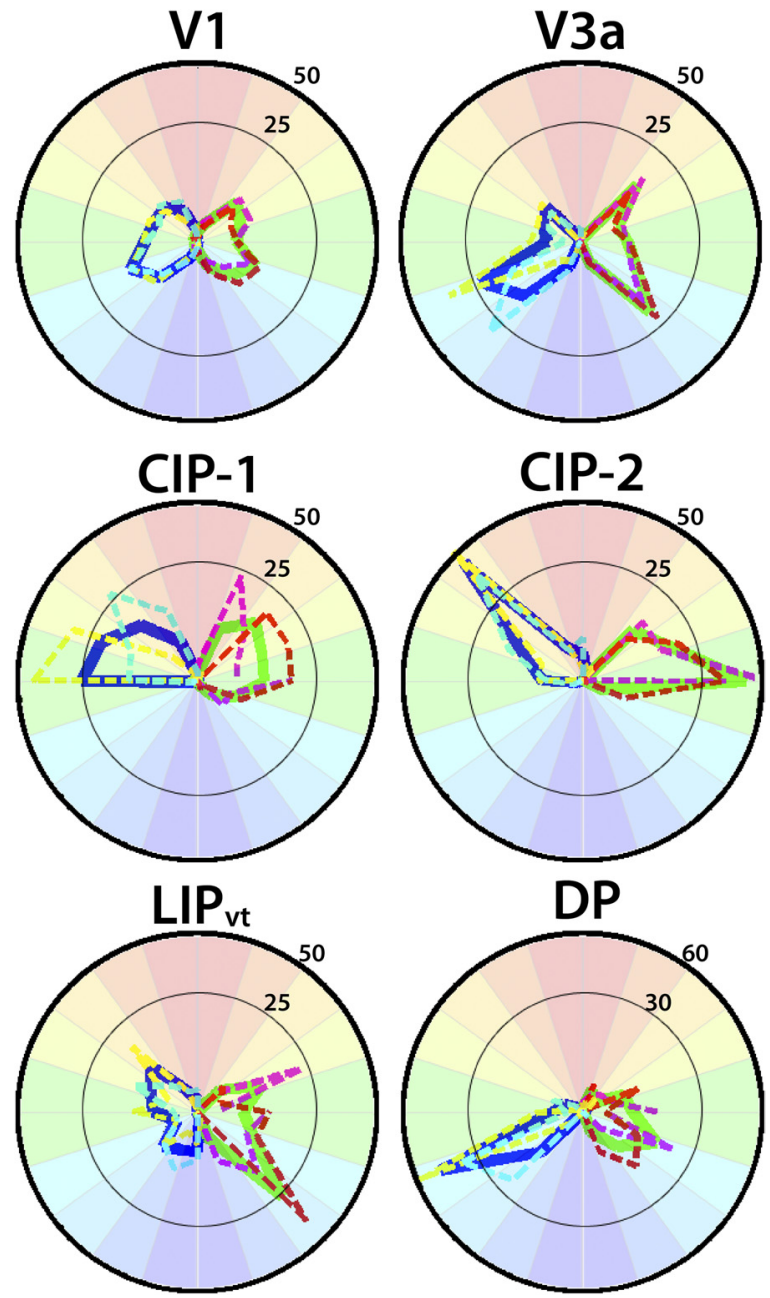

Figure 5. Visual field representations in areas V1, V3a, DP, CIP-1, CIP-2, and LIP angle plots based on polar angle maps thresholded at $2 \mathrm{~s}$ of the cycle SEM variance (see Materials and Methods). The percentage of visual field coverage within each area was calculated for both monkeys individually and then averaged. Dashed lines correspond to visual field coverage for individual monkeys (M1: yellow RH, purple LH; M2: light blue RH, red LH). The solid lines correspond to the average visual field coverage (RH: blue; LH: green). All areas almost exclusively represented contralateral visual space with little variance in visual field representation between the two monkeys.

tralateral visual space (Table 3). The caudal IPS areas, CIP-1/2, contained a larger representation of the upper quadrant. Conversely, area DP contained a larger representation of the lower quadrant. For comparison, $47 \pm 1 \%$ of nodes represented the upper quadrant and $50 \pm 2 \%$ of nodes represented the lower quadrant in V1. Due to the limited number of samples $(n=4)$, more data will be necessary to conclusively assume any asymmetries in visual field for the CIP areas or DP.

\section{Reproducibility of polar angle phase maps}

To further evaluate the consistency and reproducibility of the topography, polar angle phase estimates were determined for clockwise and counterclockwise runs separately. The resulting phase maps were highly consistent between clockwise and counterclockwise runs, as indicated qualitatively by the similarities in characteristics of polar angle phase progressions described above (supplemental Fig. 5, available at www.jneurosci.org as supplemental material). To quantify the similarity of phase estimates between clockwise and counterclockwise data on a node-by-node 
Table 4. Mean alignment index values from clockwise $(\mathrm{CW})$ and counterclockwise (CCW) runs within monkeys and from polar angle and eccentricity runs between monkeys $\mathrm{M} 1$ and $\mathrm{M} 2$ for RH and $\mathrm{LH}$

\begin{tabular}{|c|c|c|c|c|c|}
\hline & V3a & DP & CIP-1 & CIP-2 & $\mathrm{LIP}_{\mathrm{vt}}$ \\
\hline \multicolumn{6}{|c|}{ Alignment indices within monkeys } \\
\hline $\mathrm{CW}$ versus $\mathrm{CCW}$ RH & $0.95(0.01)$ & $0.95(0.01)$ & $0.91(0.04)$ & $0.92(0.01)$ & $0.90(0.01)$ \\
\hline CW versus CCW LH & $0.96(0.01)$ & $0.95(0.01)$ & $0.91(0.02)$ & $0.93(0.01)$ & $0.89(0.02)$ \\
\hline \multicolumn{6}{|c|}{ Alignment indices between monkeys } \\
\hline Polar angle RH & $0.91(0.002)$ & $0.88(0.004)$ & $0.84(0.007)$ & $0.92(0.006)$ & $0.94(0.002)$ \\
\hline Polar angle LH & $0.96(0.001)$ & $0.90(0.005)$ & $0.96(0.002)$ & $0.93(0.003)$ & $0.89(0.004)$ \\
\hline Eccentricity RH & $0.85(0.004)$ & $0.82(0.005)$ & $0.70(0.005)$ & $0.64(0.007)$ & $0.84(0.006)$ \\
\hline Eccentricity LH & $0.84(0.003)$ & $0.85(0.005)$ & $0.80(0.007)$ & $0.84(0.006)$ & $0.75(0.004)$ \\
\hline
\end{tabular}

basis, AIs were calculated for both hemispheres in both monkeys (see Materials and Methods). The AI values range from 0 , which indicates that the values obtained in the two datasets were completely out of phase by $180^{\circ}$, to 1 , which indicates perfect phase alignment. Mean AI values were averaged across monkeys for V3a, DP, CIP-1, CIP-2, and LIP (Table 4). In comparison, AI values for $\mathrm{V} 1$ ranged between 0.97 and 0.92 . Histograms of mean AIs are shown for V1, V3a, DP, CIP-1, CIP-2, and LIP (see supplemental Fig. 6, available at www.jneurosci.org as supplemental material). Additionally, the calculated correlation coefficients of each ROI were significant for each monkey's polar phase measurements (all $r$ values $>0.67$, all $p$ values $<10^{-10}$ uncorrected; median $r=0.78$ ), demonstrating that there was good alignment throughout each ROI for each monkey. These analyses provide a quantitative measure for the consistency of the topographic organization across runs regardless of the direction of stimulus rotation.

\section{Group average map}

To evaluate the consistency of the large-scale topographic organization across the individual animals, polar angle and eccentricity data from both monkeys were mapped onto a standard-mesh surface (see Materials and Methods), and average topography maps were calculated for each hemisphere. The topographic organization throughout V3a, DP, CIP-1, CIP-2, and LIP vt was remarkably similar in visual field representation as compared to that seen in individual monkeys (Figs. 1, 2, 5, 6A, B). Though the sample size is limited, this consistency across all four hemispheres of the two monkeys suggests that the organization is not much influenced by interindividual variability in the higher-order areas in the macaque brain, which is in contrast to the known individual variability of human PPC (Schluppeck et al., 2005; Silver et al., 2005; Swisher et al., 2007).

To further evaluate the similarity of phase estimates between the datasets of the two monkeys on a node-by-node basis, AI values were calculated between M1 and M2. Histograms of mean polar angle AIs are shown for V1, V3a, DP, CIP-1, CIP-2, and $\mathrm{LIP}_{\mathrm{vt}}$ in supplemental Figure 7A (available at www.jneurosci.org as supplemental material). The mean AI values for polar angle are listed in Table 4. Additionally, the calculated correlation coefficients of each ROI were significant for each monkey's polar phase measurements (all $r$ values $>0.51$, all $p$ values $<10^{-10}$ uncorrected; median $r=0.64$ ), indicating that there was good alignment throughout each ROI between monkey datasets. This analysis provides quantitative measures for the consistency of the topographic organization between individual monkeys.

The mean AI values for eccentricity are listed in Table 4. Histograms of mean eccentricity AIs are shown for V1, V3a, DP, CIP-1, CIP-2, and LIP ${ }_{\text {vt }}$ (see supplemental Fig. 7B, available at www.jneurosci.org as supplemental material). The calculated
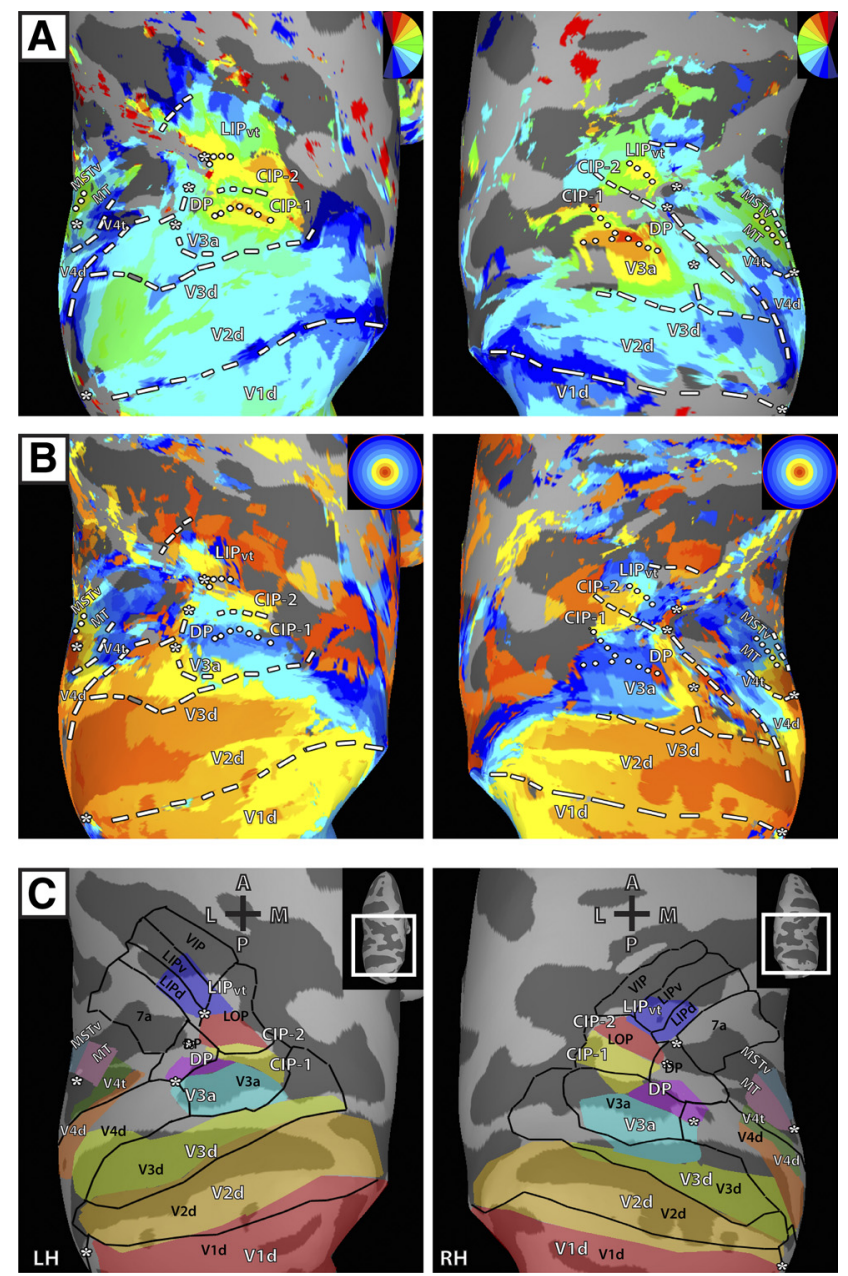

Figure 6. Average polar angle and eccentricity maps in dorsal occipital and parietal cortex on standard-mesh surfaces in comparison to the Lewis/Van Essen parcellation. Standard-mesh surfaces were created from the individual cortical surface reconstructions of each monkey (see Materials and Methods). The use of standard-mesh surfaces allowed for node-to-node correspondence across surfaces of both monkeys. $\boldsymbol{A}, \boldsymbol{B}$, All conventions and abbreviations as in Figure 1. $C$, Schematic borders of topographically defined areas (see Figs. $1 C, 2 C$ ) in conjunction with borders of visual areas from the Lewis and Van Essen parcellation (2000a,b) in the F99 macaque atlas (Van Essen, 2002) (black lines and labels).

correlation coefficients of each ROI, though smaller than those for the polar angle data, were significant for each monkey's eccentricity phase measurements (all $r$ values $>0.28$, all $p$ values $<10^{-5}$ uncorrected; median $r=0.37$ ). For comparison, AI values were also calculated for polar angle ( $\mathrm{RH} \mathrm{0.93,} r=0.72, p<$ $10^{-10}$ uncorrected; LH 0.92, $r=0.65, p<10^{-10}$ uncorrected) and eccentricity data (RH 0.92, $r=0.84, p<10^{-10}$ uncorrected; LH $0.90, r=0.74, p<10^{-10}$ uncorrected) for V1. 


\section{Comparison to anatomical parcellation of PPC}

To compare our results to an anatomical and histological parcellation of PPC, area borders (Lewis and Van Essen, 2000a,b) from the F99 macaque atlas (F99; Van Essen, 2002) were mapped onto the standard-mesh surface and compared to the boundaries determined from the average topography data of V3a, DP, CIP-1, CIP-2, and LIP ${ }_{\mathrm{vt}}$ (see Materials and Methods). Overall, there was good agreement between the atlas and topography data, particularly for early visual areas V1, V2, and V3 as well as V3a. However, there were a few notable differences between the functional mapping and the atlas data (Fig. 6C). Visuotopically defined DP overlapped with posterior portions of the F99-defined DP, but also included anterior portions of the F99-defined V3a. There was no direct correspondence of CIP-1 and CIP-2 to individual areas in the atlas. Both areas fell largely within area LOP, thereby subdividing area LOP into posterior and anterior portions, though CIP-1 also overlapped with anterior portions of the F99-defined V3a. Though CIP-2 did not overlap with F99-defined LIP, CIP-2 may also overlap with other anatomical and functional definitions of LIP (Andersen et al., 1990; Blatt et al., 1990). Visuotopically defined LIP $_{\mathrm{vt}}$ overlapped with both LIPd and LIPv of the atlas with representations of central space falling largely within LIPd, and more peripheral representations falling within LIPv. In both hemispheres, visuotopically defined LIP $_{\mathrm{vt}}$ comprised only the posterior half of LIP defined by the atlas. Even though the comparison to anatomically and histologically defined areas and zones of an atlas relative to functional topography in individual animals can only reveal approximate correspondences, the comparison of our data to the F99 parcellation schema may prove useful for investigators performing recordings in these regions, taking the atlas as a guide for electrode placement.

\section{Comparison to functional organization of human PPC}

Recent fMRI studies, including from our laboratory and others, have identified several visuotopically organized areas within dorsal visual cortex extending into the posterior parietal cortex in humans using a variety of different mapping methods including phase-encoded retinotopic mapping similar to that used here (Sereno et al., 2001; Silver et al., 2005; Schluppeck et al., 2005; Swisher et al., 2007; Konen and Kastner, 2008) (for review, see Silver and Kastner, 2009). In Figure 7, we compare the topographic organization of PPC in the macaque and human based on polar angle (Konen and Kastner, 2008) and representations of central space (Konen and Kastner, 2008, unpublished observations) (also see Swisher et al., 2007). In both species, polar angle and eccentricity phase progressions were apparent throughout the lunate sulcus and into PPC. As has been noted previously, the topographic organization of $\mathrm{V} 3 \mathrm{a}$ in the macaque is similar to area V3a in the human (Van Essen et al., 2001; Brewer et al., 2002; Fize et al., 2003; Tsao et al., 2003). In both species, there is a posterioranterior polar angle phase progression from LVM to UVM representations with a representation of central space on its lateral border. The topographic organization observed for DP appears similar to the topography of human area V3b. In both species, there is a posterior-anterior polar angle phase progression from LVM to UVM representations with a representation of the central space on its medial border shared with V3a. The topographic organization and location in relation to surrounding topographic regions of CIP-1 and CIP-2 in the macaque is similar to areas V7 (IPS-0) and IPS-1 in the human. In both species, there is a posterior-anterior polar angle phase progression starting and ending with an UVF representation. However, in the human, the border of IPS- 0 and IPS- 1 is defined by a reversal in polar phase in the
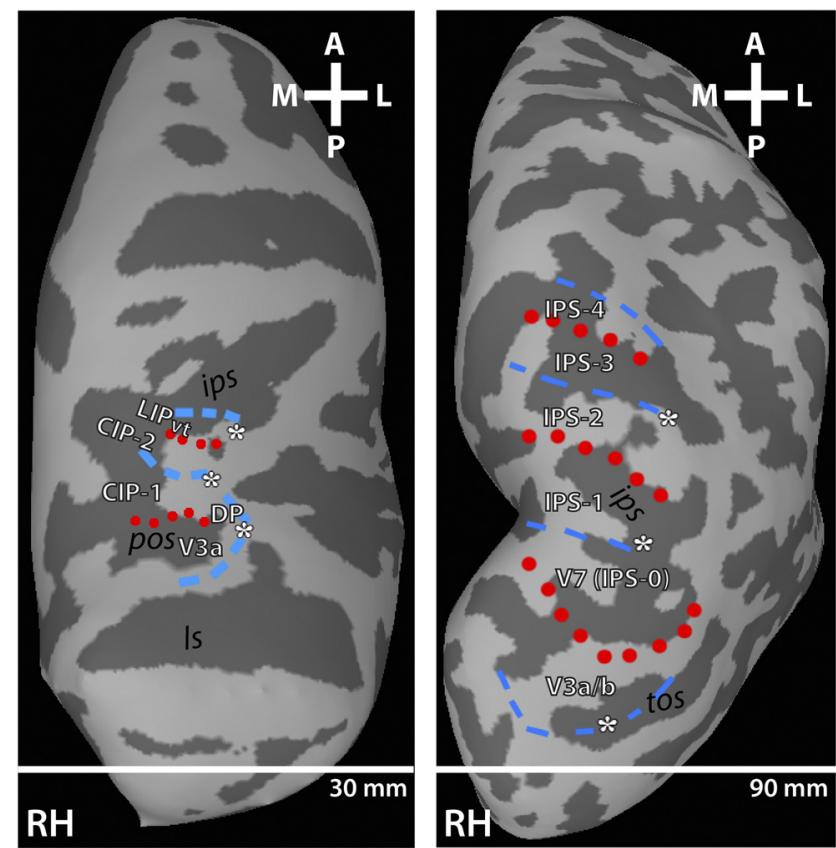

Figure 7. Comparison of dorsal visual cortex topography between monkey and human. Comparison of the fMRI-defined retinotopic organization of dorsal occipital and parietal cortex in both monkeys (left column) and humans (right column). Lines denote areal boundaries formed by phase angles at or close to the upper (red, dotted) or lower (blue, dashed) vertical meridian. Is, Lunate sulcus; pos, parieto-occipital sulcus; ips, intraparietal sulcus; tos, transverse occipital sulcus.

LVF, whereas in the monkey, the border between CIP-1 and CIP-2 is largely within the HM (though a sparse LVF representation was identified along the border in two of four hemispheres). For both monkey and human topographies, the representations of central space are located on the lateral border, with more peripheral representations located medially. $\operatorname{LIP}_{\mathrm{vt}}$ in the macaque appears similar to IPS-2 in the human. In both species, there is a posterior-anterior polar angle phase progression from UVM to LVM representations with a representation of central space on the lateral border.

Topographic organization within the IPS in humans consists of additional areas, IPS-3/4/5, extending further anterior. The IPS also extends anterior from LIP $_{\mathrm{vt}}$ in the macaque. However, consistent topographic organization was not identified beyond $\mathrm{LIP}_{\mathrm{vt}}$ in all hemispheres. It is important to note that our comparison of the topographic organization of the dorsal pathway in humans and monkeys is not aimed at establishing homology between areas, but instead, it is aimed at revealing similarities and differences in large-scale brain topography that may aid future investigations with a simple starting point to find regions that have analogous functions or regions that show similar functional organization.

\section{Discussion}

We investigated topographic organization of dorsal extrastriate cortex and PPC using fMRI and phase-encoded retinotopic mapping in monkeys trained to maintain fixation. By considering both the polar angle and eccentricity phase estimates, we identified four visuotopically organized areas representing contralateral visual space within PPC. A representation of the visual field adjacent and lateral to area V3a was found within dorsal portions of the prelunate gyrus, referred to as DP. Adjacent and anterior to V3a within the caudal, lateral portions of the IPS, two previously 
not described visuotopic areas were found, referred to as CIP-1 and CIP-2. Adjacent and anterior to CIP-2, we identified a representation of the visual field within the lateral bank of the IPS, referred to as $\operatorname{LIP}_{\mathrm{vt}}$. The visuotopic organization within dorsal extrastriate cortex and PPC and the presented framework for outlining area borders was highly consistent in all four hemispheres.

Macaque dorsal extrastriate cortex and PPC have been subdivided based on results from anatomy and physiology studies, and several different parcellation schemes have been derived (Pandya and Seltzer, 1982; Seltzer and Pandya, 1986; Colby et al., 1988; Andersen et al., 1990; Felleman and Van Essen, 1991; Preuss and Goldman-Rakic, 1991; Lewis and Van Essen, 2000a,b). We compared our data to the areas delineated in the F99 macaque atlas (Lewis and Van Essen, 2000a,b; Van Essen, 2002) since it proposes subregions within caudal IPS, dorsal prelunate, and the lateral IPS, and has been applied to both physiology (Stoet and Snyder, 2004; Huk and Shadlen, 2005; Janssen et al., 2008; Chen et al., 2010) and fMRI studies (Tsao et al., 2003; Denys et al., 2004; Durand et al., 2007; Kagan et al., 2010; Patel et al., 2010).

Within dorsal portions of the prelunate gyrus, we identified a visual field map, DP, that predominantly contained representations of the LVF with adjacent cortex within POS representing the horizontal and upper vertical meridians. Our results are consistent with physiology recordings that have found the receptive fields within DP to be concentrated within the contralateral LVF (Andersen et al., 1990), and with fMRI studies that have found representations of the LVF within the dorsal prelunate gyrus (Fize et al., 2003). Using optical imaging, Heider et al. (2005) found representations of both the UVF and LVF within DP, though the topography varied between monkeys with no apparent systematic progression of visual field locations. Thus, our results extend these prior findings by identifying a systematic visual field map within the dorsal prelunate gyrus and its relation to neighboring topographic cortex.

Within the caudal PPC, we found, to our knowledge for the first time, spatial topography suggesting that this region of cortex may be functionally subdivided into two separate areas, referred to as CIP-1 and CIP-2. CIP-1/2 were located within caudal PPC, including anterior portions of the POS and lateral IPS. CIP-2 extended into lateral portions of posterior IPS that may correspond to cortex defined as part of LIP (Blatt et al., 1990; Kagan et al., 2010). The posterior border of CIP-1 abutted the borders of V3a and DP with the anterior border of CIP-2 extending into the lateral bank of the IPS. This region of cortex has been shown to represent three-dimensional (3D) shape and texture information (for review, see Katsuyama et al., 2010). Future investigations are required to define a functional role of topographic organization in the processing of $3 \mathrm{D}$ stimulus features, and to characterize whether the response properties of CIP-2 more closely reflect previously defined CIP (Sakata et al., 1998; Taira et al., 2000) relative to LIP (Bushnell et al., 1981; Gnadt and Andersen, 1988; Shadlen and Newsome, 2001).

Within the lateral IPS, we identified a visual field map, LIP $_{\mathrm{vt}}$, that shared its posterior border with the anterior border of CIP-2 and encompassed both dorsal and ventral portions of lateral IPS. Based on results from previous physiology studies, the topographic organization within LIP has been rather unclear (Blatt et al., 1990; Platt and Glimcher, 1998; Ben Hamed et al., 2001). Blatt et al. (1990) reported a posterior-anterior gradient from LVF to UVF representations of the contralateral visual field, whereas Ben Hamed et al. (2001) reported the opposite pattern, a posterioranterior gradient from UVF to LVF representations. Both studies
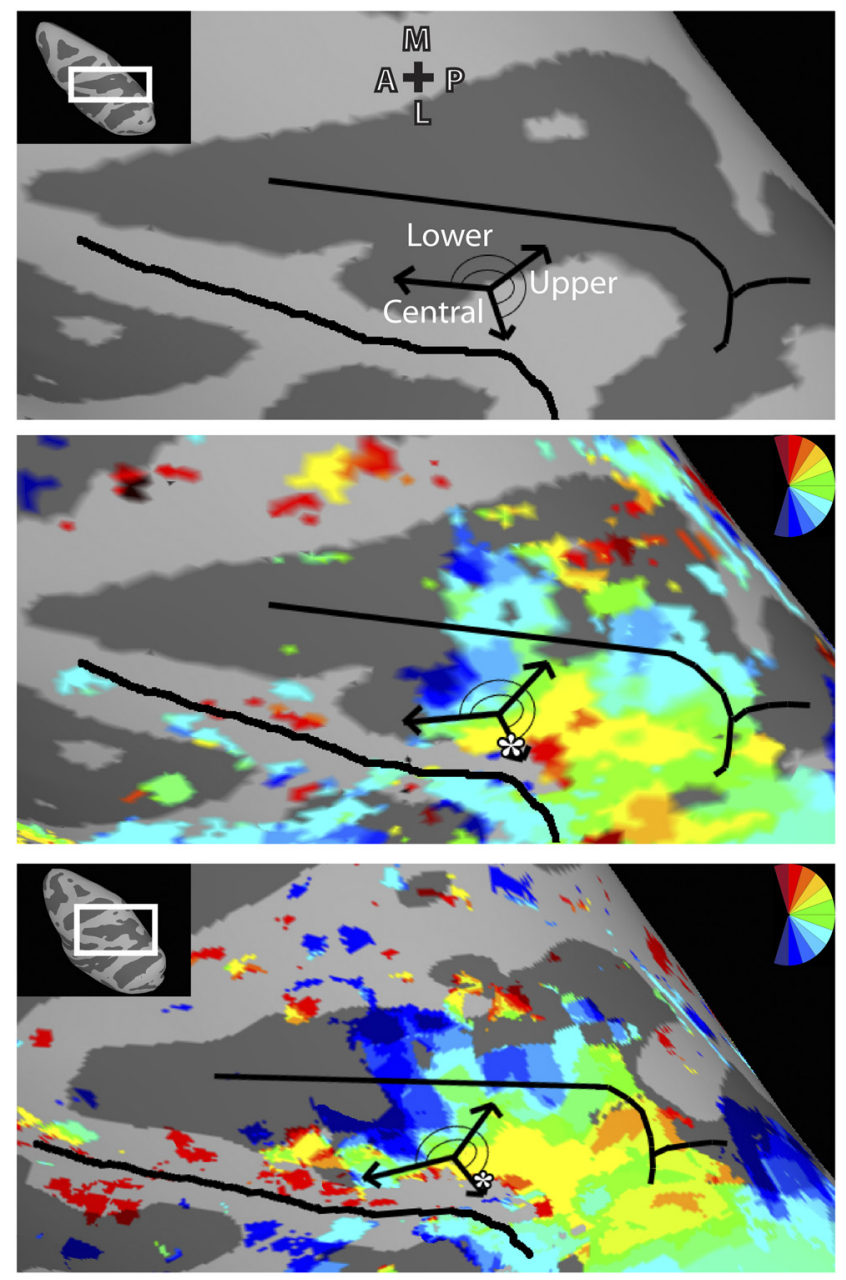

Figure 8. Comparison of LIP topography based on physiology and fMRI data. Top, Schema of LIP topography adapted with permission from Ben Hamed et al. (2001) (their Fig. 8C). The fundus of the IPS and crown of the inferior parietal lobule are denoted by black lines. A representation of the upper visual field was found in posterior LIP, and the lower visual field representation was found anterior. A representation of central space was identified on the lateral border of LIP. Middle and bottom, The schema of LIP topography defined by physiology data overlaid upon the topography of LIP from the LH of monkey M1 (middle) and the group map (bottom) revealed by fMRI. There is strong correspondence of LIP topography between the physiology and fMRI data with the upper visual field progressing anterior and medial to a lower visual field representation, and a representation of central space on the lateral border (white asterisk).

agreed regarding a central space representation within LIPd. However, a third report was unable to find any systematic organization of contralateral space within LIP (Platt and Glimcher, 1998). The polar angle phase progression of $\operatorname{LIP}_{\mathrm{vt}}$ appears in agreement with the topographic organization suggested by Ben Hamed et al. (2001), as shown in Figure 8, where we adapted the schema of LIP topography based on their single-unit data [Ben Hamed et al. (2001), their Fig. 8C] and projected it onto the LH of M1. However, the polar angle phase progression of CIP-2 just posterior to $\mathrm{LIP}_{\mathrm{vt}}$ is in good agreement with the topographic organization and anatomical coordinates reported by Blatt et al. (1990), suggesting that the discrepancies between these two physiology studies are due to differences in the recording site location and the existence of multiple organized visuotopic maps within lateral IPS. Thus, our data are in good agreement with physiology and offer an interpretation regarding discrepancies observed in previous studies. 
Our results of visuotopic organization within LIP partially confirm, but more importantly extend recent findings of topographic organization within PPC using fMRI (Patel et al., 2010) (see also Fize et al., 2003). Using a covert attention task on visual stimuli presented at only few different polar angle and eccentricity locations within the visual field, a topographic representation of the contralateral hemifield was found that appeared to be confined to LIPv (Patel et al., 2010). It was concluded that PPC contains a single topographic representation that can only be revealed in a complex cognitive task, suggesting a specific role of $\mathrm{LIPv}$ in higher cognition. While our results concur with the posterior-anterior progression of an UVF to LVF representation, there are several notable differences between our findings and those of Patel et al. (2010). First and foremost, we demonstrate a systematic and continuous progression of phase angle representations within LIP, which is an important criterion in establishing a visual field map (see Wandell et al., 2007). This requires identifying the most effective visual field position for each location within a map and can be best achieved with a continuous mapping approach such as phase-encoded mapping, rather than by stimulating a few discrete locations within the visual field. It is also noteworthy that the visuotopic map within LIP was revealed using standard mapping procedures under passive viewing conditions without requirement of a cognitive task. Second, the visuotopic map within LIP is not isolated, but is embedded in a rich topographic organization of PPC with several visuotopically organized areas adjacent to $\mathrm{LIP}_{\mathrm{vt}}$, which were differentiated on the basis of their systematic representation of visual space and reversals in polar angle phase progression at or near the vertical meridians. Third, given the posterior-anterior direction of polar angle phase progression, the dorsolateral representation of central space that was identified in our and prior physiology studies appears to be a better candidate for $\mathrm{LIP}_{\mathrm{vt}}$ than an anterior fovea reported within LIPv (Patel et al., 2010). Throughout the visual system, the progressions of polar angle and eccentricity are typically represented orthogonal (and not parallel) to each other. It is possible that the anterior representation of central space that was also found in the present study corresponds to additional topographic representations within anterior $\operatorname{LIP}_{\mathrm{v}}$.

A spatial map of saccade trajectories was recently found within anatomically defined LIPv (Savaki et al., 2010). It is not entirely clear how this map of oculomotor space relates to the visuotopic map identified in our data and prior studies due to the differences in criteria used for parcellating LIPd and LIPv (Medalla and Barbas, 2006). However, the apparent close proximity of the oculomotor map to the anterior intraparietal area [see Savaki et al. (2010), their Figs. 3b , 9, 10a] suggests that the oculomotor map lies anterior to the visuotopic map observed within the posterior half of the anatomical extent of LIP. Consistent with this interpretation, Kagan et al. (2010) reported stronger BOLD modulation for saccades toward the contralateral visual field within an anterior portion of LIP (aLIP), which appears to be anterior to LIP $_{\text {vt }}$ [see Kagan et al. (2010), their Fig. 1C and supplemental Fig. 3]. Interestingly, Savaki et al. (2010) reported representations for small saccade trajectories within LIPv, which may correspond to the representations of central visual space observed in the current data anterior to LIP $_{\mathrm{vt}}$, but within the anatomical extent of LIP. The findings of oculomotor and visuotopic maps, possibly in different parts of LIP, suggest the existence of topographic organization along multiple functional reference frames in macaque PPC.

Several visuotopically organized areas within dorsal extrastriate cortex and PPC have recently been reported using fMRI in humans (Sereno et al., 2001; Silver et al., 2005; Schluppeck et al., 2005; Swisher et al., 2007; Konen and Kastner, 2008) (for review, see Silver and Kastner, 2009). In our comparison of macaque and human large-scale PPC topography, several parallels in polar angle and eccentricity representations were identified based on the topography within individual areas and their relation to the surrounding large-scale topography. In addition to the previously noted correspondence of V3a topography, macaque CIP-1 and human IPS-0, CIP-2, and IPS-1, as well as LIP vt $_{\text {and IPS-2, }}$ showed striking similarities in topographic organization. Similarities in large-scale topographic organization between species do not necessitate or imply homology, nor do they preclude functional dissociations between species. Rather, large-scale cortical organization can be used to systematically investigate functional properties between species. Our results provide a framework for detailed comparisons of parietal cortical areas between humans and monkeys that should prove useful in relating the functional similarities and dissociations between species.

\section{References}

Andersen RA, Asanuma C, Essick G, Siegel RM (1990) Corticocortical connections of anatomically and physiologically defined subdivisions within the inferior parietal lobule. J Comp Neurol 296:65-113.

Arcaro MJ, McMains SA, Singer BD, Kastner S (2009) Retinotopic organization of human ventral visual cortex. J Neurosci 29:10638-10652.

Argall BD, Saad ZS, Beauchamp MS (2006) Simplified intersubject averaging on the cortical surface using SUMA. Hum Brain Mapp 27:14-27.

Bandettini PA, Jesmanowicz A, Wong EC, Hyde JS (1993) Processing strategies for time-course data sets in functional MRI of the human brain. Magn Reson Med 30:161-173.

Ben Hamed S, Duhamel JR, Bremmer F, Graf W (2001) Representation of the visual field in the lateral intraparietal area of macaque monkeys: a quantitative receptive field analysis. Exp Brain Res 140:127-144.

Blatt GJ, Andersen RA, Stoner GR (1990) Visual receptive field organization and cortico-cortical connections of the lateral intraparietal area (area LIP) in the macaque. J Comp Neurol 299:421-445.

Brewer AA, Press WA, Logothetis NK, Wandell BA (2002) Visual areas in macaque cortex measured using functional magnetic resonance imaging. J Neurosci 22:10416-10426.

Brewer AA, Liu J, Wade AR, Wandell BA (2005) Visual field maps and stimulus selectivity in human ventral occipital cortex. Nat Neurosci 8:1102-1109.

Bushnell MC, Goldberg ME, Robinson DL (1981) Behavioral enhancement of visual responses in monkey cerebral cortex. I. Modulation in posterior parietal cortex related to selective visual attention. J Neurophysiol 46:755-772.

Chen A, DeAngelis GC, Angelaki DE (2010) Macaque parieto-insular vestibular cortex: responses to self-motion and optic flow. J Neurosci 30:3022-3042.

Chung MK, Robbins SM, Dalton KM, Davidson RJ, Alexander AL, Evans AC (2005) Cortical thickness analysis in autism with heat kernel smoothing. Neuroimage 25:1256-1265.

Colby CL, Gattass R, Olson CR, Gross CG (1988) Topographical organization of cortical afferents to extrastriate visual area PO in the macaque: a dual tracer study. J Comp Neurol 269:392-413.

Cox RW (1996) AFNI: software for analysis and visualization of functional magnetic resonance neuroimages. Comput Biomed Res 29:162-173.

Cox RW, Jesmanowicz A (1999) Real-time 3D image registration for functional MRI. Magn Reson Med 42:1014-1018.

Dale AM, Fischl B, Sereno MI (1999) Cortical surface-based analysis. I. Segmentation and surface reconstruction. Neuroimage 9:179-194.

Daniel PM, Whitteridge D (1961) The representation of the visual field on the cerebral cortex in monkeys. J Physiol 159:203-221.

Denys K, Vanduffel W, Fize D, Nelissen K, Peuskens H, Van Essen D, Orban GA (2004) The processing of visual shape in the cerebral cortex of human and nonhuman primates: a functional magnetic resonance imaging study. J Neurosci 24:2551-2565.

Desimone R, Ungerleider LG (1986) Multiple visual areas in the caudal superior temporal sulcus of the macaque. J Comp Neurol 248:164-189.

Durand JB, Nelissen K, Joly O, Wardak C, Todd JT, Norman JF, Janssen P, 
Vanduffel W, Orban GA (2007) Anterior regions of monkey parietal cortex process visual 3D shape. Neuron 55:493-505.

Engel SA, Rumelhart DE, Wandell BA, Lee AT, Glover GH, Chichilnisky EJ, Shadlen MN (1994) fMRI of human visual cortex. Nature 369:525.

Engel SA, Glover GH, Wandell BA (1997) Retinotopic organization in human visual cortex and the spatial precision of functional MRI. Cereb Cortex 7:181-192.

Felleman DJ, Van Essen DC (1991) Distributed hierarchical processing in the primate cerebral cortex. Cereb Cortex 1:1-47.

Fischl B, Sereno MI, Dale AM (1999) Cortical surface-based analysis. II: Inflation, flattening, and a surface-based coordinate system. Neuroimage 9:195-207.

Fize D, Vanduffel W, Nelissen K, Denys K, Chef d'Hotel C, Faugeras O, Orban GA (2003) The retinotopic organization of primate dorsal V4 and surrounding areas: a functional magnetic resonance imaging study in awake monkeys. J Neurosci 23:7395-7406.

Gattass R, Gross CG (1981) Visual topography of striate projection zone (MT) in posterior superior temporal sulcus of the macaque. J Neurophysiol 46:621-638.

Gattass R, Sousa AP, Gross CG (1988) Visuotopic organization and extent of V3 and V4 of the macaque. J Neurosci 8:1831-1845.

Gattass R, Nascimento-Silva S, Soares JG, Lima B, Jansen AK, Diogo AC, Farias MF, Botelho MM, Mariani OS, Azzi J, Fiorani M (2005) Cortical visual areas in monkeys: location, topography, connections, columns, plasticity and cortical dynamics. Philos Trans R Soc Lond B Biol Sci 360:709-731.

Gnadt JW, Andersen RA (1988) Memory related motor planning activity in posterior parietal cortex of macaque. Exp Brain Res 70:216-220.

Hagler DJ Jr, Sereno MI (2006) Spatial maps in frontal and prefrontal cortex. Neuroimage 29:567-577.

Hansen KA, Kay KN, Gallant JL (2007) Topographic organization in and near human visual area V4. J Neurosci 27:11896-11911.

Heider B, Jandó G, Siegel RM (2005) Functional architecture of retinotopy in visual association cortex of behaving monkey. Cereb Cortex $15: 460-478$.

Horton JC, Hoyt WF (1991) The representation of the visual field in human striate cortex. A revision of the classic Holmes map. Arch Ophthalmol 109:816-824.

Huk AC, Shadlen MN (2005) Neural activity in macaque parietal cortex reflects temporal integration of visual motion signals during perceptual decision making. J Neurosci 25:10420-10436.

Janssen P, Srivastava S, Ombelet S, Orban GA (2008) Coding of shape and position in macaque lateral intraparietal area. J Neurosci 28:6679-6690.

Jo HJ, Lee JM, Kim JH, Shin YW, Kim IY, Kwon JS, Kim SI (2007) Spatial accuracy of fMRI activation influenced by volume- and surface-based spatial smoothing techniques. Neuroimage 34:550-564.

Jo HJ, Lee JM, Kim JH, Choi CH, Gu BM, Kang DH, Ku J, Kwon JS, Kim SI (2008) Artificial shifting of fMRI activation localized by volume- and surface-based analyses. Neuroimage 40:1077-1089.

Kagan I, Iyer A, Lindner A, Andersen RA (2010) Space representation for eye movements is more contralateral in monkeys than in humans. Proc Natl Acad Sci U S A 107:7933-7938.

Kastner S, DeSimone K, Konen CS, Szczepanski SM, Weiner KS, Schneider KA (2007) Topographic maps in human frontal cortex revealed in memory-guided saccade and spatial working-memory tasks. J Neurophysiol 97:3494-3507.

Katsuyama N, Yamashita A, Sawada K, Naganuma T, Sakata H, Taira M (2010) Functional and histological properties of caudal intraparietal area of macaque monkey. Neuroscience 167:1-10.

Kolster H, Mandeville JB, Arsenault JT, Ekstrom LB, Wald LL, Vanduffel W (2009) Visual field map clusters in macaque extrastriate visual cortex. J Neurosci 29:7031-7039.

Kolster H, Peeters R, Orban GA (2010) The retinotopic organization of the human middle temporal area MT/V5 and its cortical neighbors. J Neurosci 30:9801-9820.

Konen CS, Kastner S (2008) Representation of eye movements and stimulus motion in topographically organized areas of human posterior parietal cortex. J Neurosci 28:8361-8375.

Larsson J, Heeger DJ (2006) Two retinotopic visual areas in human lateral occipital cortex. J Neurosci 26:13128-13142.

Lewis JW, Van Essen DC (2000a) Mapping of architectonic subdivisions in the macaque monkey, with emphasis on parieto-occipital cortex. J Comp Neurol 428:79-111.

Lewis JW, Van Essen DC (2000b) Corticocortical connections of visual, sensorimotor, and multimodal processing areas in the parietal lobe of the macaque monkey. J Comp Neurol 428:112-137.

Maguire WM, Baizer JS (1984) Visuotopic organization of the prelunate gyrus in rhesus monkey. J Neurosci 4:1690-1704.

May JG, Andersen RA (1986) Different patterns of corticopontine projections from separate cortical fields within the inferior parietal lobule and dorsal prelunate gyrus of the macaque. Exp Brain Res 63:265-278.

Medalla M, Barbas H (2006) Diversity of laminar connections linking periarcuate and lateral intraparietal areas depends on cortical structure. Eur J Neurosci 23:161-179.

Pandya DN, Seltzer B (1982) Intrinsic connections and architectonics of posterior parietal cortex in the rhesus monkey. J Comp Neurol 204:196-210.

Patel GH, Shulman GL, Baker JT, Akbudak E, Snyder AZ, Snyder LH, Corbetta M (2010) Topographic organization of macaque area LIP. Proc Natl Acad Sci U S A 107:4728-4733.

Pinsk MA, Moore T, Richter MC, Gross CG, Kastner S (2005) Methods for functional magnetic resonance imaging in normal and lesioned behaving monkeys. J Neurosci Methods 143:179-195.

Pinsk MA, Arcaro M, Konen CS, Li X, Kastner S, Inati SJ (2008) Improved functional MRI of the macaque ventral visual pathway at $3 \mathrm{~T}$ using multiecho EPI and dynamic, field map corrected image reconstruction. Soc Neurosci Abstr 34:260.2.

Pinsk MA, Arcaro M, Weiner KS, Kalkus JF, Inati SJ, Gross CG, Kastner S (2009) Neural representations of faces and body parts in macaque and human cortex: a comparative FMRI study. J Neurophysiol 101:2581-2600.

Platt ML, Glimcher PW (1998) Response fields of intraparietal neurons quantified with multiple saccadic targets. Exp Brain Res 121:65-75.

Preuss TM, Goldman-Rakic PS (1991) Architectonics of the parietal and temporal association cortex in the strepsirhine primate Galago compared to the anthropoid primate Macaca. J Comp Neurol 310:475-506.

Saad Z, Reynolds R, Argall B, Japee S, Cox R (2004) SUMA: an interface for surface-based intra- and inter-subject analysis with AFNI. Proc IEEE Int Symp Biomed Imaging 2:1510-1513.

Sakata H, Taira M, Kusunoki M, Murata A, Tanaka Y, Tsutsui K (1998) Neural coding of $3 \mathrm{D}$ features of objects for hand action in the parietal cortex of the monkey. Philos Trans R Soc Lond B Biol Sci 353:1363-1373.

Savaki HE, Gregoriou GG, Bakola S, Raos V, Moschovakis AK (2010) The place code of saccade metrics in the lateral bank of the intraparietal sulcus. J Neurosci 30:1118-1127.

Schluppeck D, Glimcher P, Heeger DJ (2005) Topographic organization for delayed saccades in human posterior parietal cortex. J Neurophysiol 94:1372-1384.

Schneider KA, Richter MC, Kastner S (2004) Retinotopic organization and functional subdivisions of the human lateral geniculate nucleus: a highresolution functional magnetic resonance imaging study. J Neurosci 24:8975-8985

Seltzer B, Pandya DN (1986) Posterior parietal projections to the intraparietal sulcus of the rhesus monkey. Exp Brain Res 62:459-469.

Sereno MI, Huang RS (2006) A human parietal face area contains aligned head-centered visual and tactile maps. Nat Neurosci 9:1337-1343.

Sereno MI, Dale AM, Reppas JB, Kwong KK, Belliveau JW, Brady TJ, Rosen BR, Tootell RB (1995) Borders of multiple visual areas in humans revealed by functional magnetic resonance imaging. Science 268:889-893.

Sereno MI, Pitzalis S, Martinez A (2001) Mapping of contralateral space in retinotopic coordinates by a parietal cortical area in humans. Science 294:1350-1354.

Shadlen MN, Newsome WT (2001) Neural basis of a perceptual decision in the parietal cortex (area LIP) of the rhesus monkey. J Neurophysiol 86:1916-1936.

Silver MA, Kastner S (2009) Topographic maps in human frontal and parietal cortex. Trends Cogn Sci 13:488-495.

Silver MA, Ress D, Heeger DJ (2005) Topographic maps of visual spatial attention in human parietal cortex. J Neurophysiol 94:1358-1371.

Stoet G, Snyder LH (2004) Single neurons in posterior parietal cortex of monkeys encode cognitive set. Neuron 42:1003-1012.

Swisher JD, Halko MA, Merabet LB, McMains SA, Somers DC (2007) Visual topography of human intraparietal sulcus. J Neurosci 27:5326-5337.

Taira M, Tsutsui KI, Jiang M, Yara K, Sakata H (2000) Parietal neurons 
represent surface orientation from the gradient of binocular disparity. J Neurophysiol 83:3140-3146.

Tsao DY, Vanduffel W, Sasaki Y, Fize D, Knutsen TA, Mandeville JB, Wald LL, Dale AM, Rosen BR, Van Essen DC, Livingstone MS, Orban GA, Tootell RB (2003) Stereopsis activates V3A and caudal intraparietal areas in macaques and humans. Neuron 39:555-568.

Van Essen DC (2002) Surface-based atlases of cerebellar cortex in the human, macaque, and mouse. Ann N Y Acad Sci 978:468-479.

Van Essen DC (2004) Organization of visual areas in macaque and human cerebral cortex. In: The visual neurosciences (Chalupa L, Werner JS, eds), pp 507-521: MIT Press.

Van Essen DC, Zeki SM (1978) The topographic organization of rhesus monkey prestriate cortex. J Physiol 277:193-226.

Van Essen DC, Maunsell JH, Bixby JL (1981) The middle temporal visual area in the macaque: myeloarchitecture, connections, functional properties and topographic organization. J Comp Neurol 199:293-326.

Van Essen DC, Newsome WT, Maunsell JH (1984) The visual field representation in striate cortex of the macaque monkey: asymmetries, anisotropies, and individual variability. Vision Res 24:429-448.

Van Essen DC, Lewis JW, Drury HA, Hadjikhani N, Tootell RB, Bakircioglu M, Miller MI (2001) Mapping visual cortex in monkeys and humans using surface-based atlases. Vision Res 41:1359-1378.

Wandell BA, Dumoulin SO, Brewer AA (2007) Visual field maps in human cortex. Neuron 56:366-383.

Zeki S (1980) The representation of colours in the cerebral cortex. Nature 284:412-418.

Zeki SM (1969) Representation of central visual fields in prestriate cortex of monkey. Brain Res 14:271-291. 